\title{
A Diagnostic Human Workload Assessment Algorithm for Collaborative and Supervisory Human-Robot Teams
}

\author{
JAMISON HEARD, Vanderbilt University \\ RACHEL HEALD, University of Kansas \\ CAROLINE E. HARRIOTT, Vanderbilt University \\ JULIE A. ADAMS, Oregon State University
}

High-stress environments, such as first-response or a NASA control room, require optimal task performance, as a single mistake may cause monetary loss or even the loss of human life. Robots can partner with humans in a collaborative or supervisory paradigm to augment the human's abilities and increase task performance. Such teaming paradigms require the robot to appropriately interact with the human without decreasing either's task performance. Workload is related to task performance; thus, a robot may use a human's workload state to modify its interactions with the human. Assessing the human's workload state may also allow for dynamic task (re-)allocation, as a robot can predict whether a task may overload the human and, if so, allocate it elsewhere. A diagnostic workload assessment algorithm that accurately estimates workload using results from two evaluations, one peer based and one supervisory based, is presented. The algorithm correctly classified workload at least $90 \%$ of the time when trained on data from the same human-robot teaming paradigm. This algorithm is an initial step toward robots that can adapt their interactions and intelligently (re-)allocate tasks.

CCS Concepts: • Computing methodologies $\rightarrow$ Neural networks; $\bullet$ Computer systems organization $\rightarrow$ Robotic autonomy;

Additional Key Words and Phrases: Human-robotic interaction, workload assessment, human-robot teaming

\section{ACM Reference format:}

Jamison Heard, Rachel Heald, Caroline E. Harriott, and Julie A. Adams. 2019. A Diagnostic Human Workload Assessment Algorithm for Collaborative and Supervisory Human-Robot Teams. ACM Trans. Hum.-Robot Interact. 8, 2, Article 7 (May 2019), 30 pages.

https://doi.org/10.1145/3314387

\section{INTRODUCTION}

Changing the ways that robots interact with humans in uncertain, dynamic, and high-intensity environments (i.e., first response or a NASA control room) is needed to realize effective human-robot teams. Dynamic domains require new innovative human-robot teaming methodologies, which are

This work was partially supported by National Science Foundation award CNS 1659746, NASA Cooperative Agreement Number NNX16AB24A, AFOSR Award FA9550-09-1-0108, NSF Grant IIS-0643100, and ONR Award N00014-12-1-0987.

Authors' addresses: J. Heard and C. E. Harriott, Vanderbilt University, 2201 West End Ave, Nashville, TN 37235; emails: jamison.r.heard@vanderbilt.edu, ceharriott@gmail.com; R. Heald, University of Kansas, 1450 Jayhawk Blvd, Lawrence, KS 66045; email: rachelmary15@ku.edu; J. A. Adams, Oregon State University, 1680 SW Monroe Ave, Corvallis, OR 97331; email: julie.a.adams@oregonstate.edu.

Permission to make digital or hard copies of all or part of this work for personal or classroom use is granted without fee provided that copies are not made or distributed for profit or commercial advantage and that copies bear this notice and the full citation on the first page. Copyrights for components of this work owned by others than the author(s) must be honored. Abstracting with credit is permitted. To copy otherwise, or republish, to post on servers or to redistribute to lists, requires prior specific permission and/or a fee. Request permissions from permissions@acm.org.

(C) 2019 Copyright held by the owner/author(s). Publication rights licensed to ACM.

2573-9522/2019/05-ART7

https://doi.org/10.1145/3314387 
adaptive in nature, due to varying task demands. These methodologies require mechanisms that can drive the robot's interactions, such that the robot provides valuable contributions to achieving the task, while appropriately interacting with, but not hindering, the human. The human's workload state can be used to determine robot interactions that may augment team performance, due to the relationship between workload and task performance [17,55]. The overall research objective is to use physiological signals to derive human workload states that can be incorporated into future adaptive teaming systems that target adaptations or (re-)allocate tasks intelligently to improve or manage team performance.

Capturing physiological signals that are sensitive to workload in dynamic environments has become feasible, due to advances in wearable sensors. These signals respond to variations in one or more workload components (e.g., cognitive, physical, visual, speech, and auditory), which compose a human's overall workload state [37, 38]. Workload assessment algorithms use machine-learning techniques to produce workload estimates from physiological signals [24] but typically assess a variant of overall workload or simply cognitive workload. Assessing a single workload component does not provide an understanding of the specific task components contributing to the current workload state. The dynamic nature of human-robot teams requires discriminating between workload components, as tasks will vary in relation to their workload composition. True adaptive teaming systems that change their interactions or intelligently (re-)allocate tasks will require a workload assessment algorithm capable of estimating overall workload and its components.

First-response robots can use diagnostic workload information to determine how to interact with a human responder. For example, if a robot must communicate an important message, then the envisioned adaptive system's activity recognition can determine that the human is triaging a victim requiring immediate care. Knowledge of the task allows the diagnostic workload assessment algorithm to identify that the human's overload state is caused by the physical, cognitive and visual workload components. If the message to be communicated to the human is of low priority, then the robot can delay communication until it projects the triage to be completed. If the message requires immediate action, then the robot can identify another human with which to communicate.

The presented diagnostic workload assessment algorithm is the first component of the envisioned adaptive human-robot teaming system. Data from two user evaluations was used to train the algorithm, which estimates values for the cognitive and physical workload components, while a workload model is used for the remaining components. Future work will estimate the speech and auditory workload components. An overall workload state is derived from the five workload components. The algorithm's ability to classify workload, generalize across populations, humanrobot interaction paradigms and tasks were evaluated using the data from the human-robot experiments.

Section 2 provides background information, while Section 3 presents the workload assessment algorithm. Section 4 describes the experimental design for the teaming evaluations. Section 5 presents three algorithmic analyses and discussion. Finally, Section 6 concludes the manuscript.

\section{RELEVANT WORK}

Workload can be defined as the ratio of resources required to achieve tasks to the resources the human has available to dedicate to the task [55]. An overload workload state occurs when the task requires a large amount of resources, but the human has a small amount of resources available to dedicate to the task. Likewise, an underload workload state occurs when a task demands a small amount of resources but the human has a large amount of resources available. Both workload states can be detrimental to task performance. The workload assessment algorithm literature does not focus on detecting the underload state; however, there are domains where an adaptive teaming system must detect the underload state (e.g., nuclear power plant control rooms). These domains 
Table 1. Workload Metrics by Overall Workload and Its Associated Components [23]

\begin{tabular}{|c|c|c|c|c|c|c|c|}
\hline Metric & Response & $\mathbf{W}_{\mathbf{O}}$ & $\mathbf{W}_{\mathrm{C}}$ & $\mathbf{W}_{\mathbf{A}}$ & $\mathbf{W}_{\mathbf{S}}$ & $\mathbf{W}_{\mathbf{V}}$ & $\mathbf{W}_{\mathbf{P}}$ \\
\hline Heart Rate Variability (HRV) [32,54] & decreases & & & & & & \\
\hline Heart Rate (HR) $[18,32]$ & increases & & & & & & \\
\hline Respiration Rate (RR) $[33,47]$ & decreases & & & & & & \\
\hline Skin Temperature (ST) $[39,40]$ & decreases & & & & & & \\
\hline Noise Level (NL) $[8,21]$ & increases & & & & & & \\
\hline Postural Magnitude (PM) $[20,43]$ & increases & & & & & & \\
\hline Posture Sway (PS) $[20,35]$ & increases & & & & & & \\
\hline
\end{tabular}

Note: $\mathbf{W}_{\mathrm{O}}=$ Overall Workload, $\mathbf{W}_{\mathrm{C}}=$ Cognitive Workload, $\mathbf{W}_{\mathrm{A}}=$ Auditory Workload, $\mathbf{W}_{\mathrm{S}}=$ Speech Workload, $\mathbf{W}_{\mathrm{V}}=$ Visual Workload, $\mathbf{W}_{\mathbf{P}}=$ Physical Workload.

have long periods of low workload followed by bursts of high workload. It is critical that the humans remain vigilant during low-workload periods to better respond in high-workload conditions, but the underload state can reduce vigiliance [55]. Thus, an adaptive teaming system must be able to detect and adapt to both overload and underload workload states.

Workload assessment algorithms for real-world human-robot teams must rely on objective metrics, typically physiological, to determine a human's workload state. A number of objective metrics, including some that suffer signal degradation during long-duration and high-mobility tasks, correlate to changes in overall workload and the associated workload components. The metrics used for the presented algorithm and the corresponding correlations (gray cells) are shown in Table 1. The visual workload column is empty, as none of the workload metrics correlate to visual workload. Workload metrics not incorporated in the algorithm are not shown in the table. Cardiovascular measures (e.g., heart rate and heart-rate variability $[1,32,54])$ and brain activity measures (e.g., electroencephalography (EEG) [42, 51, 52] and functional near-infrared spectroscopy (FNIRS) [25, 26]) are sensitive to cognitive workload. EEG and FNIRS are sensitive to movement [9], which hinders their viability for dynamic domains. Eye-tracking measures (e.g., fixation duration and pupil dilation $[5,9,36])$ are sensitive to visual workload but may not be viable for dynamic nonstationary scenarios, as some eye-tracking metrics require known focus regions. Pupil dilation and blink rate do not require known focus regions; however, the metrics are greatly confounded by light and fatigue, respectively. Speech-response time and noise level are indicators of auditory workload [6, 8, 21], while speech workload can be assessed using speech-based measures (e.g., speech-response time, speech rate, and filler utterances [6, 30]). Heart rate [18, 32], respiration rate $[33,47]$, skin temperature $[39,40]$, and posture-based metrics (e.g., posture sway and posture magnitude $[20,35,43])$ correlate to physical workload. Physiological metrics vary from individual to individual (i.e., physical fitness impacts heart rate); thus, workload assessment algorithms must account for these individual differences to generalize across populations [11, 24].

The state-of-the-art workload assessment algorithms use the workload metrics as features to classify workload (e.g., References [3, 27, 45, 53, 56]). Rusnock et al. [48] used a model tree classifier to estimate overall workload based on EEG, pupil dilation, blink rate, fixation duration, heart rate, heart-rate variability, and respiration rate. The classifier was trained on an IMPRINT Pro overall workload model and accurately assessed overall workload. A limitation is that the algorithm cannot generalize across populations and is unable to discriminate among different workload components, which limits its generalizability across human-robot interaction paradigms.

Durkee et al. [14] developed an algorithm to estimate each subscale of the NASA Task Load Index (NASA TLX) [22]. NASA TLX's static subscale estimates were perturbed with noise to generate a continuous workload model. The algorithm used EEG, heart rate, heart-rate variability, and pupil 
dilation to estimate each workload model and generalized across populations. A limitation of the algorithm is that it does not generalize across human-robot interaction paradigms, as it does not estimate speech and auditory workload. Additionally, the algorithm estimates subjective workload, which can be confounded by the participant and is not an objective workload measure.

Individual proprietary algorithms classified cognitive, visual, auditory, speech, and physical workload [44]. The algorithms used EEG, heart rate, heart-rate variability, respiration rate, head movement, and electromyography signals to classify workload levels. The approach detects changes in the workload component states, but these results have not been composed into an overall workload state. Not assessing overall workload limits the algorithm's viability for adaptive teaming systems, as the system will not identify overload and underload conditions.

No existing algorithm discerns both the overall workload state and each workload component contributing to the overall workload state [24]. However, an adaptive teaming system needs to understand the state of each workload component to appropriately target adaptations to a specific workload component. Further, the existing algorithms are unable to generalize across populations and human-robot interaction paradigms. The diagnostic workload assessment algorithm has been designed to address these challenges.

\section{WORKLOAD ASSESSMENT ALGORITHM}

The diagnostic workload assessment algorithm is designed to estimate overall workload and each workload component every 30s using knowledge of the task being completed. This time threshold or window size is due to heart rate and heart-rate varability requiring at least $30 \mathrm{~s}$ of data to be highly sensitive to workload variations $[9,10]$. Other window sizes may increase or decrease algorithmic performance, but a single size was chosen for simplicity. Future work will investigate the impact of window size on the algorithm's performance. The desired estimates are based on a workload model, which is generated using IMPRINT Pro [4].

Prior work [23] developed a similar algorithm, which relied on physiological metrics (see Table 1) to estimate cognitive and physical workload and subjective surveys to estimate the remaining workload components. Overall workload was calculated using a weighted aggregation of the workload components, where the weights were determined by IMPRINT Pro workload models. The previous algorithm is incapable of estimating workload in real time due to the reliance on subjective surveys. The current algorithm incorporates more sophisticated filtering techniques, does not rely on subjective metrics, uses IMPRINT Pro workload models as the desired workload component estimates, aggregates mapped physiological metrics using multi-variate regression, and uniformly aggregates the workload component estimates into an overall workload estimate. The new algorithm can be used in real time, which is not evaluated in this manuscript.

IMPRINT Pro creates models of complex task networks that designate start and stop times for each task and anchors each task to workload component values (i.e., a conversation is anchored to a speech workload component value of 4.0). The task networks and workload component values are used to derive continuous models across seven workload components: auditory, cognitive, visual, speech, gross motor, fine motor, and tactile. The presented models combine the gross motor, fine motor, and tactile components into the physical workload model. An overall workload model is generated by uniformly aggregating the workload component models.

Although IMPRINT Pro generates workload models, the workload models represent predicted workload outcomes, are static, and do not adjust in real time to the current situation. Additionally, there is uncertainty between the IMPRINT Pro models and the human's actual task, which creates a mismatch between the IMPRINT Pro workload values and the human's actual workload. Using physiological metrics as the foundation of the workload assessment algorithm reduces the uncertainty and provides a more objective workload estimate. The IMPRINT Pro cognitive and physical 
workload models are simply used to train and validate the algorithm. The IMPRINT Pro auditory, speech, and visual workload models are used to estimate the model's corresponding workload components, due to a lack of objective data. A future algorithm version will use objective metrics to estimate the auditory, speech, and visual workload components.

\subsection{Adaptive Exponential Smoothing}

The algorithm's incorporated metrics and their associated workload response are listed in Table 1. Each metric is filtered using Yin and Zhang's adaptive exponential smoothing technique [56], which extends the exponential smoothing formula by adaptively choosing $\lambda$ based on shifts in the data. The exponential smoothing formula, given in Equation (1), incorporates $s_{k}$ representing the new value for the respective workload metric at time $k, \lambda$ representing a tuning parameter, and $x_{k-i}$ representing the workload metric's time series,

$$
s_{k}=(1-\lambda) s_{0}+\lambda \sum_{i=0}^{k-1}(1-\lambda)^{i} x_{k-i} .
$$

The value of $\lambda$ is determined by

$$
\lambda= \begin{cases}\lambda_{1}, & \text { if }\left|x_{k}-s_{k-1}\right| \geq a \sigma \\ & \&\left|x_{k+1}-s_{k-1}\right| \geq a \sigma \\ \lambda_{2}, & \text { otherwise. }\end{cases}
$$

A large value $\left(\lambda_{1} \geq 0.6\right)$ is chosen when a step-shift in the data exists, represented by a constant (a) multiplied by the standard deviation $(\sigma)$. Otherwise, a small value is chosen $\left(\lambda_{2} \leq 0.3\right)$. The constant $a$ is set to 1 to adaptively choose $\lambda$, based on whether the change is greater than the standard deviation of the data segment. A 30s window is applied to each filtered metric, which are then mapped to the same value range to support aggregation.

\subsection{Metric Mappings}

Neural networks map the average of each workload metric to the same range, where each metric has its own corresponding neural network. The neural network output is essentially a noisy estimate of an IMPRINT Pro workload component model and each network was trained using the ADAM optimization algorithm [34]. The neural networks were coded using the TFLearn python library [13], which builds upon Google's TensorFlow framework [2]. Each neuron's activation function was the TANH function. The metrics that correlate to only cognitive or physical workload (e.g., heart-rate variability) use a single neuron in each neural network layer (a 1-1-1-1 network) that outputs a noisy estimate of the metric's corresponding workload component. The metrics that correlate to cognitive and physical workload, e.g, heart rate, each use a 3-5-5-1 network. The additional inputs to the 3-5-5-1 networks represent the cognitive and physical task composition, which is determined by the respective IMPRINT Pro cognitive and physical workload models' value at time $t$ divided by the overall workload model value at time $t$ (i.e., CognitiveTaskComposition = CognitiveWorkloadModel $(t) /$ OverallWorkloadModel $(t)$ ). The number of neurons per hidden layer for each network were chosen to minimize the mean-squared error loss function. The each neural network output is combined according to workload component to estimate each component.

\subsection{Workload Equations}

The neural network outputs are subjected to a weighted aggregation that results in a workload value for each workload component, where the weights are determined by multi-variate 
regression. The cognitive workload, $W_{C}$, and physical workload, $W_{P}$, equations are provided in Algorithm 1 on lines 8 and 9, respectively. There are instances where posture sway (PS) and posture magnitude (PM) provide no insight into the human's physical workload level (i.e., no torso flexing occurs). Thus, two sets of weights are generated: one that includes posture sway and magnitudes (when $>0$ ) and one that does not. The auditory, $W_{A}$ and visual workload, $W_{V}$ components are estimated using their respective IMPRINT Pro models [4], as no metrics (Table 1) correlate with said components. While noise level and respiration rate correlate to speech workload, they cannot be used to determine if a human is speaking. Therefore, speech workload, $W_{S}$ is provided by IMPRINT Pro.

The uniform aggregation of the individual workload components results in the overall workload, $W_{O}$ estimate. The estimated overall workload value is mapped to one of $n$ workload states, based on thresholds determined by the IMPRINT Pro workload models used to train the algorithm. Subjective ratings are not used for determining these thresholds. For example, if three IMPRINT Pro workload models representing the underload, normal load, and overload workload states are used to train the algorithm, then the potential thresholds may be 10 and 50 . Any value less than 10 is mapped to the underload state, any value greater than 50 represents the overload state, and remaining values represent the normal load state. However, there may be a different number of workload states in other scenarios, i.e., only low- and high-workload states.

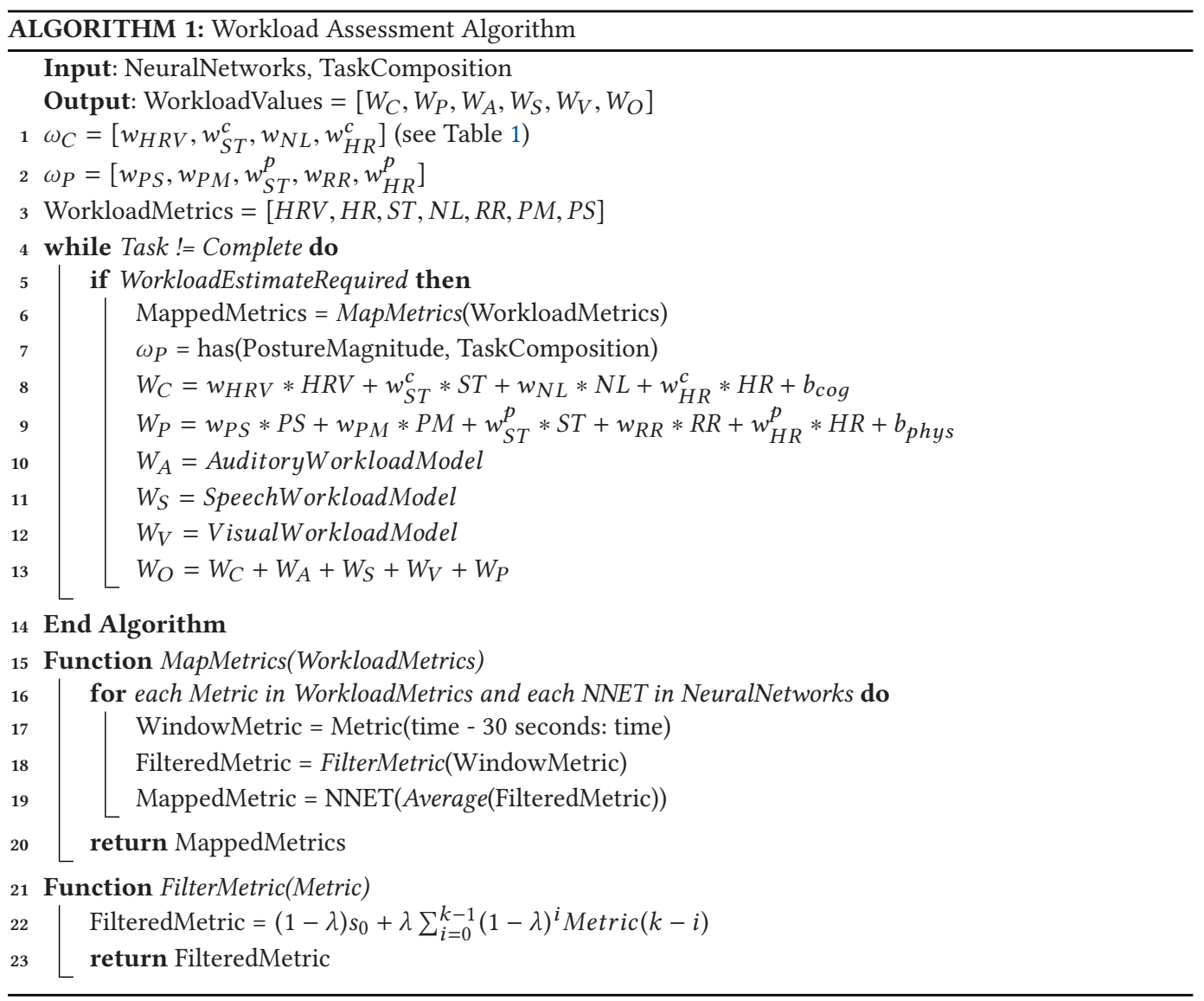




\subsection{Formal Algorithm}

Algorithm 1 combines the filtering, metric mappings, and workload estimation into a single algorithm, where $\omega$ in lines 1 and 2 represents a tuple of metric weights. The algorithm cycles through the while loop (lines 4-13) until no workload estimate is required. The algorithm generates a workload estimate by filtering the objective metrics using the MapMetrics function (lines 15-20), which applies a 30s epoch on line 17, filters the metric using the adaptive exponential smoothing formula on line 18, and applies a neural network to the smoothed data on line 19. The workload component and overall workload values are generated on lines 8-13.

\section{EXPERIMENTAL DESIGN}

Physiological data from two human-machine teaming evaluations were used to analyze the workload assessment algorithm. Both evaluations manipulated workload and used a Biopac BioHarness and wireless microphone to collect the objective metrics (see Table 1). The Biopac Bioharness chest-strap [31] collects heart rate, heart-rate variability, respiration rate, skin temperature, and posture-based metrics. All independent variables were modeled using IMPRINT Pro. Each workload condition was verified using performance and subjective metrics.

A 5-minute break occurred between each evaluation's tasks and trials. Five minutes is a common time period used with physiological measures [46], since within 5 minutes of the highest-workload task, heart rate drops significantly to within 0.6 beats per minute of baseline values. Demographic data, such as age, gender, caffeine consumption, education level, and subjective stress levels, were collected at the start of each evaluation, and no relevant interactions were identified. A power analysis determined the number of required participants. There is a difference in sample sizes between the evaluations, but the difference is accounted for in the algorithmic analysis, as the same number of data points per evaluation were use for testing and evaluating the algorithm.

\subsection{Peer-based Evaluation}

The peer-based evaluation analyzed differences in workload and task performance in humanrobot peer-based teams $[19,23]$. The results were suitable for analysis of the workload assessment algorithm. The peer-based evaluation scenario required training the participants as a civil support team member and focused on indentifying suspicous items, searching for hazardous materials and collecting samples of both liquid and solid hazardous materials. The tasks were ordered in the general order of response steps to a disaster event and partnered a robot with a human [28].

The repeated-measures design used the responder partner (human and robot) and the tasks as within-subjects elements. The participants completed four tasks with both a human partner $(\mathrm{H}-$ $\mathrm{H}$ ) and a robot partner (H-R). Two sessions were completed, one with each partner, in which, all four tasks were completed. The first task, the photo search task, involved determining whether photographs contained hazardous or suspicious items. The second task, the item search task, involved searching a hallway for hazardous or suspicious items. The third task, the solid contaminant sampling task, required participants to follow a hazard collection protocol for solid samples [29]. The liquid contaminant sampling task followed a similar hazard collection protocol to the third task but adapted for liquid hazards. The tasks were performed in the same order for each participant and team pairing. Each participant received a randomized combination of low- and high-task-workload levels. The evaluation partner presentation was counterbalanced; thus, half of the participants completed the tasks first with the human partner and next with the robot.

4.1.1 Participants. Eighteen participants completed the evaluation. Four male and five female participants worked with the human partner first (human partner first group) and five male and four female participants were paired with the robot first (robot partner first group). 
The mean age of the human partner first group was 22.63 (Standard Deviation (St. Dev.) = 6.16), with a range from 18 to 39, and the robot partner first group mean age was 21.89 (St. Dev. $=5.04$ ) with a range from 18 to 34 . The human partner first group rated their search and rescue experience on a Likert scale from 1 (little or no experience) to 9 (very experienced) as a median of 1 , with a range from 1 to 9 , while the robot partner first group rated their experience with a median of 4.5 with a range from 1 to 7 . The human partner first group rated their robotics experience on the same scale as a median of 1 , with a range from 1 to 9 , while the robot partner first group rated their experience as a median of 3 with a range from 1 to 8 . No significant difference existed between the two groups.

The human partner first group slept a median of 6.5 hours (range: 3 to 8 ) the night before their first session, while the robot partner first group slept 8 hours (range: 7 to 10) and slept significantly more hours, (Wilcox test, $U=8.5, p=0.012$ ). The human partner first group was awake for a median of 7.13 hours (range: 1 to 12.5) before the first session, while the robot partner first group was awake for 7.63 hours (range: 0.25 to 12.45 ). No significant differences existed.

The human partner first group slept a median of 6 hours (range: 3 to 9) the night before their second session, while the robot partner first group slept 7.5 hours (range: 3 to 9). The human partner first group was awake for a median of 8 hours (range: 5 to 13) before the second session, while the robot partner first group was awake for 8 hours (range: 2 to 10). No significant difference existed.

4.1.2 Metrics. The objective metrics included physiological responses (i.e., heart rate, respiration rate, heart rate variability, vector magnitude, steps taken), timing measures (i.e., subtask time, primary task response time, primary task reaction time, primary task failure rate, secondary task failure rate, a memory recall task), and the calculation of task density.

Participants were assigned a secondary task to monitor a walkie-talkie for incoming messages from the Incident Commander for their team, Team 10. An example of a message was, "Incident Command to Team 10: there is a suspicious person running south on Anderson Road." The participant was responsible for recognizing that the message was directed at Team 10 and repeating it to his or her partner when the communication arrived. The failure rate counted each time the participant did not report the message to his or her partner, and a partial failure was identified when the participant was able to respond to the Team 10 message but relayed the message content incorrectly. During low-workload tasks, there were eight total messages; two messages were for Team 10. During high-workload tasks, 6 of the 24 messages were directed to Team 10.

Subjective measures were collected but are not reported in this manuscript.

4.1.3 Environment. All tasks were located close to one another on the same floor of an academic building at Vanderbilt University. Training took place in a small office with minimal distractions, in which the photo search task also occurred. The item search task occurred in the hallway where random people were able to walk through the environment. Sound traveled into the area from nearby offices, classrooms, and laboratories. The solid contaminant sampling task occurred in an engineering laboratory, isolated from foot traffic, and contained engineering equipment, lab benches, tables, and tools. The liquid contaminant sampling task occurred in a virtual reality laboratory with two large tables on which the task area was focused.

4.1.4 Apparatus. During the H-H condition, a second experimenter acted as the human teammate. A script, on a tablet computer, dictated verbal interactions between the participant and experimenter. The same male experimenter, who wore a reflective vest, was partnered with all participants. 
Participants were instructed that the robot moved and spoke autonomously. The Pioneer 3-DX robot partner's navigation was controlled by an experimenter using line-of-sight teleoperation and a web-cam streaming video to the experimenter's laptop, unbeknownst to the participants. The robot spoke using a digital voice through on-board speakers. Participants donned a wireless microphone headset to amplify their voices when communicating with the robot. The same experimenter that controlled the robot, heard participant questions and responses, and used this knowledge to advance the robot's pre-programmed speech script. The robot's speech script was identical to the H-H condition's verbal script. When participants asked questions, the experimenter chose from a set of pre-programmed responses, repeated the robot's last statement, or wrote in a custom response.

4.1.5 Procedure. Participants completed the evaluation sessions ( $\mathrm{H}-\mathrm{H}$ and human-robot $(\mathrm{H}-\mathrm{R}))$ on different days. The mean days between participants' sessions was 13.25 (St. Dev. $=10.77$ ), where some second sessions were delayed due to participant cancellations and scheduling restrictions.

Upon arrival for the first session, participants completed a consent form and demographic questionnaire. The participants received an evaluation task briefing and were shown a 3-minute 40s training video. Participants donned the Bioharness heart rate monitor, a Looxcie wearable video camera, the Shure microphone headset, a walkie talkie with ear piece, the Fitbit activity monitor, the Scosche Rhythm+ heart rate monitor, and a reflective vest. Participants were introduced to the responder partner, either human or robot, and began the first task, the photo search task.

The photo search task required identifying suspicious items in photographs of an area taken by a surveillance team. The participant was told that a team of robots previously entered the building to photograph rooms and areas that may contain victims, hazardous chemicals, suspicious items, or nothing to investigate. The participant used an Google Nexus 7 tablet computer running the Android mobile operating system to view, search, and edit the photographs. The incident commander, a remotely located experimenter, was responsible for sending photograph folders to the participant's tablet and notes from the investigation team to the responder.

The small office space included two adjacent tables and two chairs. During the H-H teaming condition, the human partner sat at the table on the right, while the participant sat at the table to the left. During the H-R teaming condition, the robot drove to a spot near the table on the right. Participants held the tablet during the task without constraint. No stand was used to prop the tablet on the table. The participant was able to swivel the chair to face the partner, but no movement outside of controlling the tablet was required.

The participant's tablet computer received the transmitted folders of photographs via a document sharing service, Box. Periodically, new folders containing three photographs each, appeared to be investigated. Once the participant reviewed the photograph and identified something suspicious, it was his or her job to edit the photograph in the photo-editing application, Aviary, by circling the item or adding a note to describe why the item must be investigated by a follow-up team. An instruction sheet provided information regarding how to use Box and perform the Aviary photo-editing steps.

Participants performed a training session using test photographs prior to starting the 15-minute task and used the instruction sheet to learn how to use the tablet, Box application, and photoediting system without time pressure. The participant took as long as he or she desired to train on three test photos and to ask questions during the training.

Once the 15-minute task began, two sets of three photographs were searched in the lowworkload condition, and four sets of three photographs were searched in the high-workload condition. The photographs in each folder showed different angled perspectives of rooms, which included areas of the building in which the evaluation took place, such as study areas, classrooms, 


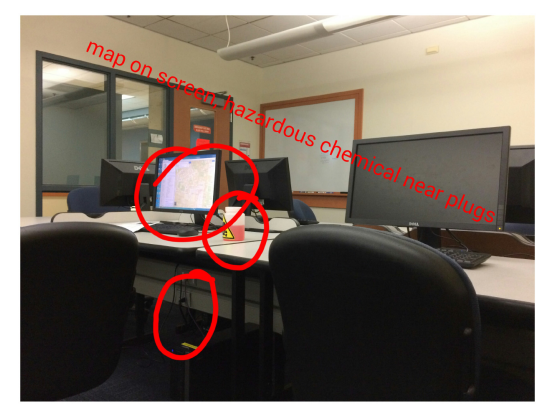

Fig. 1. Post-edited photograph for task 1.

and a computer laboratory. The folders were presented in the same order for all participants within a workload condition, but different and comparable sets of photographs were used for the $\mathrm{H}-\mathrm{H}$ and $\mathrm{H}-\mathrm{R}$ sessions. An example photograph with post-participant annotations is provided in Figure 1.

A remotely located evaluator uploaded the photographs to the participant's folder visible by the participant at predefined times. During both workload conditions, the first photo set was uploaded immediately before the task began. During the low-workload condition, the second set of photographs was uploaded 7 minutes and 30s into the task, while in the high-workload condition, the four folders arrived with 3 minutes and $45 \mathrm{~s}$ in between.

Some participants took longer to finish examining the folders. If a participant was not finished with Folder 1 by the time Folder 2 arrived, then they simply opened Folder 2 when they finished Folder 1. If the team finished evaluating Folder 1 and before Folder 2 arrived, then the responder explained that the incident commander uploads folders as soon as photographs become available. An audible beep tone was used (the same tone in all tasks) to indicate when a new folder arrived. Teams viewed the photographs individually; thus, the beeps indicated time pressure for teams that were completing the task slowly. The teams who finished before the next folder arrived were able to know precisely when the new folder arrived and start the investigation immediately.

The second task, the item search task, required conducting an exhaustive search of an environment for potentially hazardous items, while gathering environmental context and air samples. The goal was to identify all hazardous items, while searching as much of the assigned area within the time limit. The participant's role was to locate and photograph the items. The participants wore equipment to simulate personal protective equipment, including safety gloves, goggles, a dust mask, and a 10-pound backpack. The dust mask and backpack represented a civil support team's rebreather. The participant in Figure 2 is wearing the backpack, dust mask, goggles, and gloves.

This higher physical activity task involved walking around a hallway. The participants searched areas above the robot's sensors' field of view, while the human or robot partner scanned for hazards near the ground, collected air samples, and alerted the participant if any hazards or high air samples were detected. The team collaborated by discussing whether the detected items were suspicious.

Four items were investigated in the low-workload condition, while eight items were investigated in the high-workload condition. Each of the items investigated in the first session were identical, regardless of the participant's partner. A similar set of items, placed in different locations in the same environment, was used for all participants during the second session, independent of the assigned partner. The first session items are depicted in Figure 3, where the starred items were only used in high-workload tasks. The items used for the second session are depicted in Figure 4 with the same denotation for the additional high-workload items.

Either teammate determined whether the team needed to stop to investigate an item, though the responder teammate only did so when the participant missed an item that required investigation. 


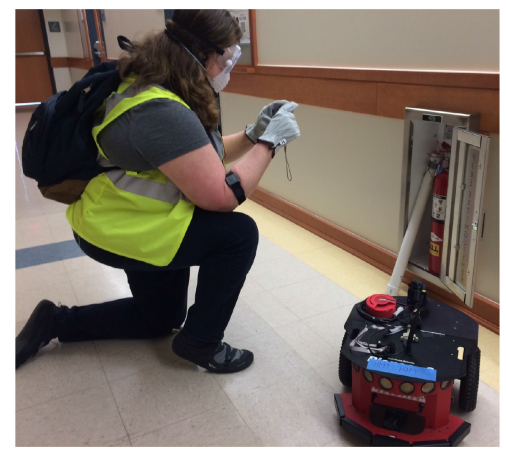

Fig. 2. Participant performing the item search task.
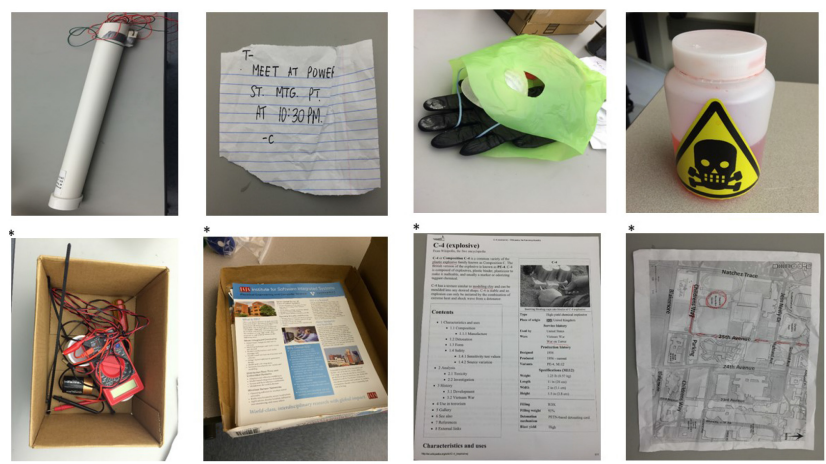

Fig. 3. The first session item search task items. Starred items $\left({ }^{*}\right)$ were only used in the high-workload condition. From top left, clockwise: a pipe bomb, cryptic note, bag with gloves and dust mask, hazardous liquid, suspiciously marked map of Vanderbilt campus, papers regarding C4 explosive use, box of advertisements (not suspicious), and a cardboard box filled with wires and suspicious material.
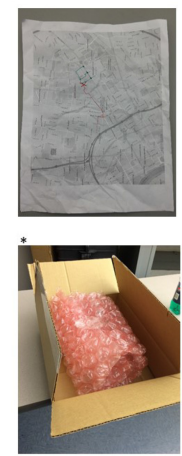
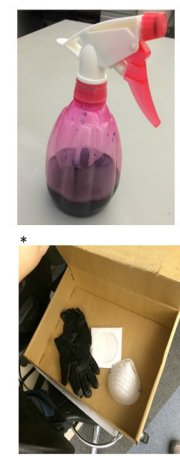
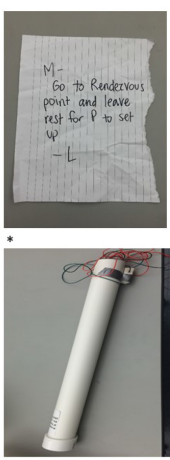
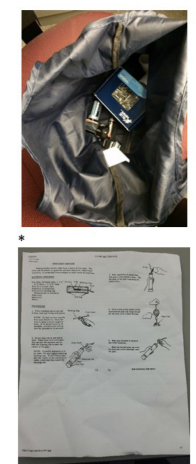

Fig. 4. The search task's second session items. Starred items ( $\left.{ }^{*}\right)$ represent the high-workload condition. From top left, clockwise: suspiciously marked map of Nashville, suspicious liquid in spray bottle, cryptic note, bag filled with batteries and nails, papers instructing the fabrication of pipe bombs, pipe bomb, box with gloves suspicious envelope with white powder, and bubble wrap (not suspicious). 


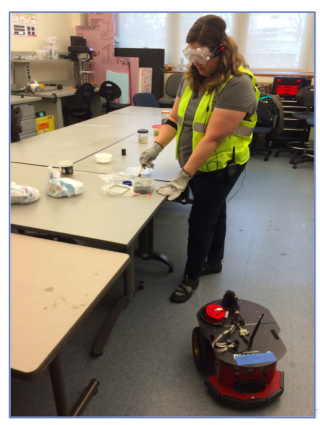

Fig. 5. An H-R team sampling solid contaminants.

During the task, the responder paused the search to send current information to incident command and waited for a response indicating approval to continue the search. Incident command's messages were indicated with an audible beep at pre-defined times (i.e., 3:45, 7:30, 11:15, and 15:00). Teams completed the investigation of items in varying time lengths; thus, the beep created time pressure for the slower teams. The teams who finished before the next message arrived were able to know precisely when the new notification was received.

The solid contaminant sampling task, the third task, required collecting samples from potentially hazardous solids in a room. The participant donned safety gloves and goggles. This task had a lower physical activity level, because the items were in close proximity and the participant did not wear the weighted backpack. The participants collected samples of the solids stored in various containers using a sterile collection kit by following guidelines audibly provided by the responder partner. These guidelines included detailed procedures that required strict compliance for maintaining safe and sterile sampling procedures. These evaluation steps were based on published government standards for the bulk sample collection of visible powders and suspected biological agents [29]. The partner indicated which solid to sample based on a message from the incident commander.

The partner provided hazard collection instructions and requested information from the participant regarding each potential hazard. Figure 5 depicts a participant completing this task.

The team entered the room with hazards visible on a table. The hazards were containers (e.g., clear plastic storage container, glass jar, and a film canister) filled with unknown, colored solids (colored sand and baby powder). Participants used sampling kits, transported in a large gardening wagon, to collect small samples from subset of the hazards in the order requested by the incident commander. Each kit contained two sandwich-sized zip-lock plastic bags, one 4-ounce glass sample jar, one stainless steel scoopula, and one alcohol wipe, all placed in a gallon-sized storage bag and wrapped in a diaper to maintain sterility and protection from breakage. The wagon contained mailing labels to seal the bags and one permanent marker for writing the time on the seal. The participants were free to move the wagon, which was stationed at the room's entrance in the same place for each session.

Two samples were assigned in the low-workload condition, and four samples were assigned in the high-workload condition. The collection steps are presented in Figure 6. The messages regarding additional samples were marked by an audible beep, which added time pressure for slower teams.

The liquid contaminant sampling task required following hazardous material sample collection procedures that dictate conducting the collection from the least hazardous materials to the most hazardous materials [29]. The participants sampled liquids, while wearing safety gloves, goggles, a 


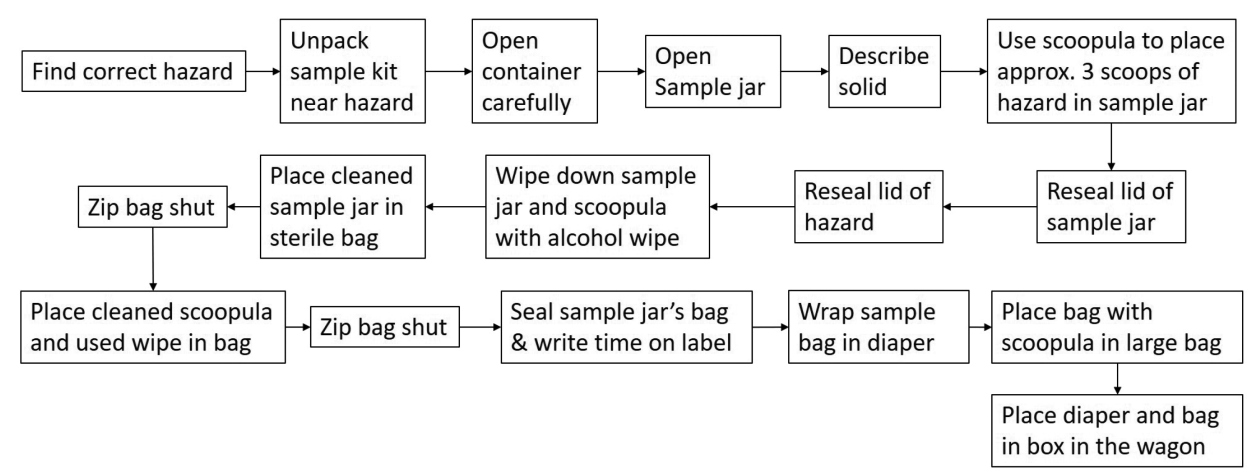

Fig. 6. The steps completed for each contaminant sample collected in the solid contaminant sampling task.

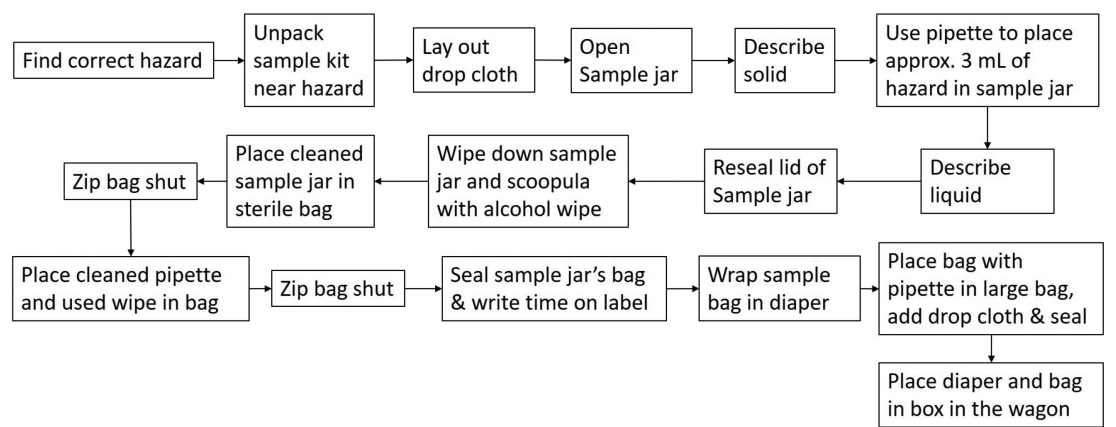

Fig. 7. The steps completed for each liquid contaminant sample.

dust mask, and the 10-pound weighted backpack, simulating the cumbersome personal protective gear (see Figure 2). This task had higher physical activity, as it required walking around a larger laboratory space between the samples, and wearing the backpack. The gloves and mask added extra physical workload by increasing the task difficulty, such as opening plastic bags.

The sampling steps were similar to the solid contaminant sampling task. The highly structured protocol, based on government requirements, ensured sterile and safe collection of the "toxic liquid hazards." The partner provided guidelines and gathered information from the participant.

Participants entered the room with two tables, each with containers (e.g., sports water bottle, glass jar, Pepsi bottle) containing liquids (water dyed with various hues of food coloring). Nine containers were set out for all tasks in the same configuration for each evaluation during both sessions. The first session assigned a different subset of the nine containers than the second session.

The collection kits contained two sandwich-sized zip-lock plastic bags, one four-ounce glass sample jar, one plastic pipette, one drop cloth cut from plastic sheeting (approximately 2 feet by 1 foot), and one alcohol wipe. The drop cloth was placed under the sampling area to catch potential spillage. The kits were stored in a gallon-sized plastic bag and wrapped in a diaper to maintain sterility and protection from breakage. The kits were stored in the gardening wagon stationed near the room's entrance. The wagon also held the mailing labels for sealing the sample and a marker for labeling it. Participants were able to move the wagon at their discretion.

The liquid contaminant collection steps are presented in Figure 7. The participant and responder roles were similar to that in the solid contaminant sampling task. Two samples were assigned in the low-workload condition, while four samples were assigned in the high-workload condition. Beeps indicated when a new sample request arrived and added time pressure to slower teams. 
After all four tasks, the participants returned for the second session, completing a questionnaire regarding their prior night's sleep, donning the equipment, and completing the evaluation in the identical task order as the first session, independent of responder partnering-human or robot.

\subsection{Supervisory-based Evaluation}

The supervisory-based evaluation focused on workload and task performance for supervision of a remotely-piloted aircraft using the NASA Multi-Attribute Task Battery (MATB-II) [12]. Four concurrent tasks are incorporated into the NASA MATB-II: tracking, system monitoring, resource management, and communication monitoring. The tracking task required using a joystik to track an object. The system monitoring task required intervening when an error occurred within the remotely piloted aircraft, while the resource management task required monitoring and maintaining the aircraft's fuel levels. The communication task required responding to air traffic control requests.

Each participant completed three 15-minute trials designed to elicit one of three workload states: underload, normal load, and overload. The evaluation is a repeated-measures design, with the workload states as the within-subjects element. Participants were randomly assigned to one of six trial orderings to counter-balance the workload conditions and reduce ordering effects.

Supervisory control $[15,50]$ is defined as human supervising robot(s) by specifying the robots' actions, monitoring task completion, and intervening if need be. Each participant read a script prior to training on the NASA MATB-II to stage the supervisory scenario. The script detailed that the participant was monitoring an unmanned aerial vehicle surveying an area. If the vehicle found a target, then the participant was to control the tracking of the target (tracking task). Errors occurred in the form of gauges and lights, which the participant was instructed to fix (system monitoring task). Participants were told to monitor and maintain the vehicle's fuel levels (resource management task) as well as respond to communications (communication monitoring task).

4.2.1 Participants. The 30 participants (18 female and 12 male) had a mean age of 25.70 (St. Dev. $=8.65$ ), with an age range from 18 to 62 . Video game experience may impact participants' performance on the NASA MATB-II, due to the multi-tasking nature of the task environment. Twenty-five participants played video games for three or less hours per week. Participants indicated that they exercise a mean of $3.86($ St. Dev. $=1.59)$ times per week. Seventeen participants did not drink any caffeine the day of the experiment, while six drank $\leq 16 \mathrm{oz}$. and seven drank $\geq 16 \mathrm{oz}$.

The participants slept an average of $6.58($ St. Dev. $=1.57)$ hours the night before the first day of the experiment and an average of 6.78 (St. Dev. $=1.85$ ) hours two nights prior. The participants' stress levels rated on a Likert scale $(1=$ little to $9=$ extreme $)$ and were rated on an average of 2.90 (St. Dev. = 1.76), while fatigue levels were rated on average of 2.83 (St. Dev. $=1.32)$ on the same scale. A Kruskall-Wallis test found no significant effect of sleep, fatigue, or stress on workload.

4.2.2 Metrics. Objective and subjective workload measures were collected throughout the evaluation. The BioHarness measured heart rate, respiration rate, skin temperature, body activity, and posture (via a three-dimensional accelerometer). Noise level was collected using a decibel meter. Heart rate variability was derived from the BioHarness's ECG signal.

The NASA MATB-II automatically collected performance metrics of the tasks. The tracking task's performance was measured by the error in pixels between the center of the cross-hairs and the center of the object, which was collected every $15 \mathrm{~s}$. The system monitoring task's performance was determined by response time and failure rate. Response time was the number of seconds a participant took to click on a light or gauge once the respective light or gauge goes out of range, while failure rate represented the number of out of range lights and gauges that were not corrected (i.e., clicked) within 15s. The resource management task's performance was determined by the 


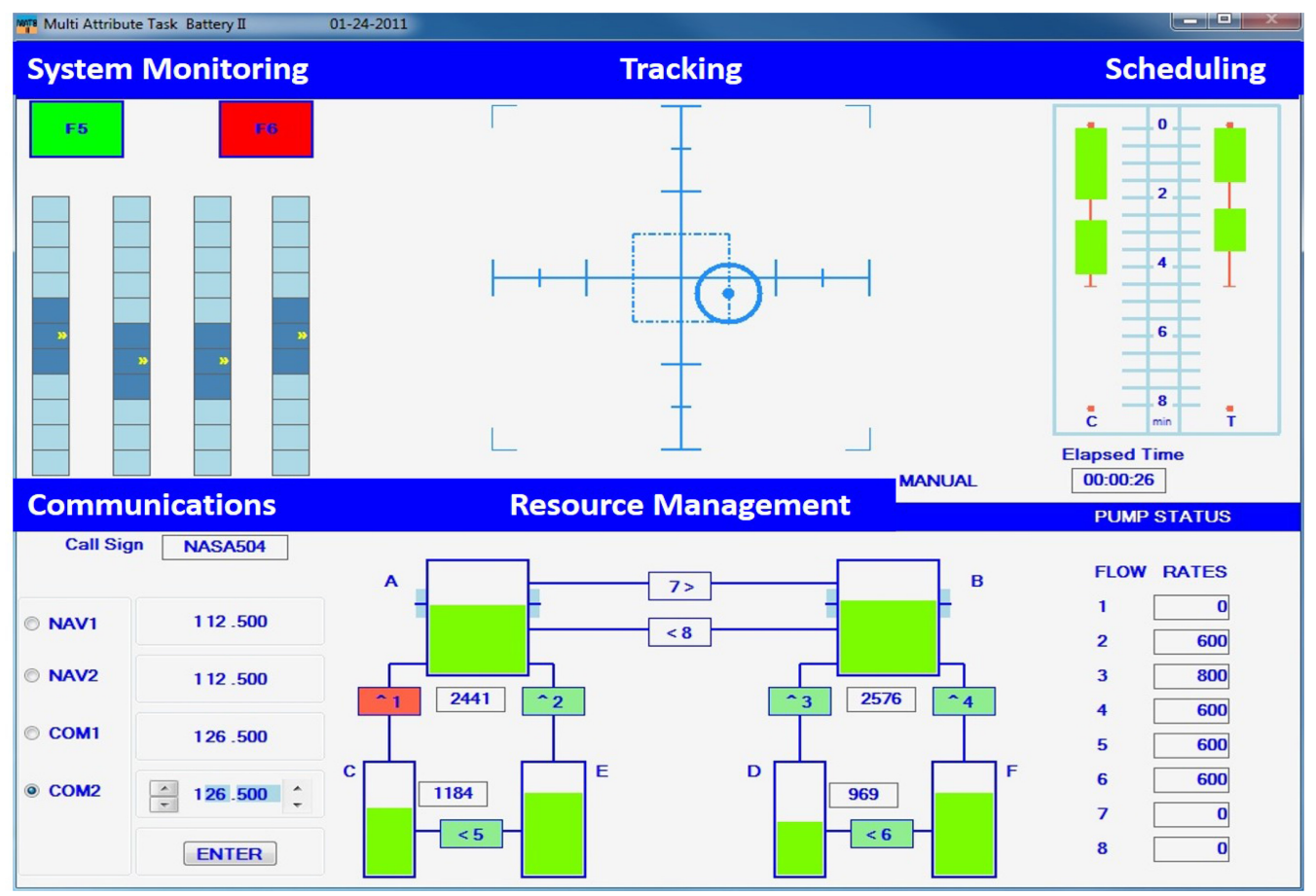

Fig. 8. The NASA MATB-II task environment.

time fuel Tanks A and B were out of range (i.e., the fuel levels were not between 2,000 and 3,000 units). The fuel levels of each tank were collected every 30s. The number of failed communication requests (i.e., the participant failed to respond or the number of times the radio was tuned to the wrong frequency), determined the communications task performance.

4.2.3 Environment. The evaluation occurred in a faculty office at Vanderbilt University's campus. The participants sat in front of a single computer monitor, using a mouse and a joystik to interact with the NASA MATB-II. The experimenter sat behind and to the side of the participant.

4.2.4 Procedure. Participants completed a consent form and a demographic questionnaire upon arrival. The participants were fitted with the BioPac Bioharness and a microphone before reading a script to stage the scenario. A 10-minute training session occurred before starting the first trial.

The tracking task, depicted in the top, center of Figure 8, required participants to keep the circle with a blue dot in the middle of the cross-hairs using a joystick. Workload was manipulated by setting the tracking mode: automatic (low) and manual (high). The underload condition used the automatic mode, with no input, for the entire trial, while the overload condition required the manual mode, or full control, for the entire trial. The normal workload condition switched between manual and automatic modes approximately every 2.5 minutes, which was determined using IMPRINT Pro. The text in the lower-right corner of the tracking task area indicated task mode.

The system monitoring task required monitoring two colored buttons and four gauges, shown in the upper left of Figure 8. If the green button turned gray (off) or the other button turned red (on), then the value was out of range and required resetting by selecting the button. The four gauges have a randomly moving indicator, up and down, that typically remained in the middle. 
Participants clicked on a gauge if it was out of range (i.e., the indicator was too high or too low). The underload condition had 1 out-of-range instance per minute, overload had 20 instances per minute, and normal load had 5 instances per minute.

The resource management task included six fuel tanks (A-F) and eight fuel pumps (1-8), shown in the bottom center of Figure 8. The arrow by the fuel pump's number indicated the direction fuel was pumped. Participants were to maintain the fuel levels of Tanks A and B by turning the fuel pumps on or off. Fuel Tanks $\mathrm{C}$ and $\mathrm{D}$ had finite fuel levels, while Tanks $\mathrm{E}$ and $\mathrm{F}$ had an infinite fuel supply. A pump turned red when it failed. Zero pumps failed during the underload condition, while two or more pumps failed during the overload condition. The normal load condition switched from zero pumps failing to one or two pumps failing every minute.

The communications task required listening to air-traffic control requests for radio changes. A communication request may be "NASA 504, please change your COM 1 radio to frequency 127.550." The original MATB communications task required no speech, but a required verbal response was added. A response may be "This is NASA 504 tuning my COM 1 radio to frequency 127.550." Participants were to change the specified radio to the specified frequency by selecting the desired radio and using arrows to change the radio's frequency, as depicted in the lower left of Figure 8. Communications not directed to the participants' aircraft, as indicated by the call sign, were to be ignored. The underload condition contained fewer than or equal to two requests, the overload contained more than or equal to eight, and normal load contained two to eight requests per minute.

\section{ALGORITHM ANALYSIS}

The algorithm analysis was performed from three different perspectives: workload assessment algorithm, task generalizability, and cross-interaction paradigms. The workload assessment algorithm analysis focused on the algorithm's ability to classify workload and generalize across populations and human-robot teaming paradigms, while the task generalizability analysis determined whether the algorithm generalizes across peer-based tasks. The cross-interaction paradigm analysis focused the impact of including peer-based human-human teaming data during training on assessing workload for peer-based human-robot teams.

The algorithm was trained in a supervised fashion, where the inputs were the physiological data from time $(t-30)$ to time $(t)$ and the expected output is the corresponding IMPRINT Pro workload model value. No subjective ratings were used in the training and validation of the algorithm. The algorithms' estimates were compared to the corresponding IMPRINT Pro model workload values using descriptive statistics, while the Kruskal-Wallis test determined if the algorithms' estimates differed between workload conditions. Classification accuracy determines if the algorithm can accurately detect workload states and was calculated by dividing the number of correctly classified data points by the total number of data points. Pearson's correlation analysis analyzed the algorithms' ability to track workload changes within and across workload conditions.

\subsection{Workload Assessment Algorithm Evaluation}

Three different datasets were used to separately train the algorithm, resulting in three trained algorithms: the peer-based evaluation's data (PEER), the supervisory-based evaluation's data (SUP), and a combination of both evaluations' data (BOTH). The PEER dataset consisted of 12 participants from only the human-robot teaming condition, (70\% of the peer-based human-robot data), and the SUP dataset consists of 14 participants, (50\% of the supervisory-based data). The participants for each set were chosen at random. The different number of participants differed due to equating the total number of data points used to train the algorithm variants, as more training data potentially increases performance and creates an unbalanced comparison. The BOTH dataset 
Table 2. Model Ranges and Thresholds by Workload Condition and Component, Evaluation, and Overall Workload

\begin{tabular}{lllll}
\hline Evaluation & Condition & Cognitive & Physical & Overall \\
\hline \multirow{2}{*}{ Peer } & Low (L) & $0.00-9.35$ & $0.00-11.71$ & $0.00-32.25$ \\
& High (H) & $0.46-8.78$ & $0.93-11.77$ & $10.97-30.35$ \\
\hline Threshold & L-H & 4.39 & 5.88 & 16.12 \\
\hline \multirow{2}{*}{ Supervisory } & UL & $1.00-2.03$ & $0.00-0.70$ & $4.00-9.27$ \\
& NL & $4.93-11.63$ & $4.04-6.86$ & $13.80-39.19$ \\
& OL & $21.20-22.76$ & $11.20-12.50$ & $59.20-66.81$ \\
\hline Threshold & UL-NL & 3.48 & 2.37 & 11.53 \\
\hline
\end{tabular}

Note: $\mathrm{UL}=$ Underload, $\mathrm{NL}=$ Normal Load, $\mathrm{OL}=$ Overload.

aggregated the PEER and SUP datasets, which allowed aggregation for determining whether a single trained algorithm may be used in both human-robot teaming paradigms.

Each trained algorithm was evaluated using the respective test dataset, which included four peer evaluation participants (30\% of the peer-based data) and six supervisory evaluation participants (16\% of the supervisory-based data). The workload assessment algorithm was also trained on 20 supervisory-based participants and tested on the other 10 participants, creating a 70/30 split for training and testing datasets. The results for this algorithm variant, while not presented in this manuscript, are virtually identical to the SUP algorithm's performance and are available online. ${ }^{1}$

Thresholds for classifying the workload states were extrapolated from the IMPRINT Pro workload models and differ by evaluation. The supervisory evaluation thresholds were the mid-points between the maximum and minimum values for the condition pairs, underload-normal load and normal load-overload, respectively. Overlap existed between the peer-based evaluation's conditions; thus, the threshold is the median between the minimum low-workload value and the maximum high-workload value. The minimum and maximum values for each workload condition and the thresholds between each condition by evaluation are presented in Table 2 . The IMPRINT Pro model descriptive statistics for the peer and supervisory evaluations are found in Tables 3 and 7 , respectively.

Classification accuracy determines how well the workload assessment algorithm discriminates between workload conditions and generalizes across populations. Hypothesis $\mathrm{H}_{1}$ predicts that the algorithm will correctly classify workload states for each workload component and overall workload at least $80 \%$ of the time. This hypothesis tests the algorithm's population generalizability, as the testing set contains data from participants that are not in the training set. Classification accuracy is used to examine the algorithm's ability to generalize across human-robot teaming paradigms; however, it is expected that accuracy will decrease when the algorithm is not trained on the teaming relationship specific data. Thus, hypothesis $\mathrm{H}_{2}$ predicts that the algorithm will correctly classify workload states for each workload component and overall workload at least $70 \%$ of the time, when not trained on the human-robot teaming relationship's specific data. The algorithm's ability to track workload shifts is an important factor for workload prediction and estimation, particularly for a system intended to adapt to the human's current and projected workload state. Hypothesis $\mathrm{H}_{3}$ predicts that the algorithm's estimates will positively and significantly correlate with the IMPRINT Pro workload models, as a positive significant correlation indicates tracking of a workload shift.

The results for the three algorithms (SUP, PEER, BOTH) are presented by teaming evaluation.

\footnotetext{
${ }^{1}$ https://drive.google.com/open?id=0BxxMR5GvXYlBVEIzOVNwWlB6REk.
} 
Table 3. Workload Modeled and Algorithm Estimated Value's Descriptive and Kruskall-Wallis Statistics for the Peer Evaluation

\begin{tabular}{lc|c|c|c}
\hline \multirow{2}{*}{ Workload } & \multirow{2}{*}{ Training } & \multicolumn{3}{|c}{ Workload Condition } \\
\cline { 3 - 5 } & & Low & High & $\chi^{2}$ \\
\hline \multirow{4}{*}{ Cognitive } & Model & $3.58(2.84)$ & $6.14(1.59)$ & $61.75^{*}$ \\
\cline { 2 - 5 } & SUP & $7.61(5.62)$ & $13.25(5.20)$ & $49.12^{*}$ \\
& PEER & $3.77(2.82)$ & $5.95(1.40)$ & $35.92^{*}$ \\
& BOTH & $4.33(3.22)$ & $7.74(3.62)$ & $59.75^{*}$ \\
\hline \multirow{4}{*}{ Physical } & Model & $4.17(2.63)$ & $5.56(2.14)$ & $17.85^{*}$ \\
\cline { 2 - 5 } & SUP & $7.07(4.93)$ & $8.55(3.34)$ & 2.44 \\
& PEER & $4.28(2.50)$ & $5.35(1.89)$ & $10.94^{*}$ \\
& BOTH & $4.36(2.54)$ & $5.95(2.02)$ & $22.58^{*}$ \\
& Model & $14.33(7.83)$ & $21.31(3.36)$ & $56.26^{*}$ \\
\cline { 2 - 5 } Overall & SUP & $21.26(11.68)$ & $31.41(7.30)$ & $45.24^{*}$ \\
& PEER & $14.64(7.62)$ & $20.91(2.25)$ & $45.60^{*}$ \\
& BOTH & $15.27(7.61)$ & $23.29(4.71)$ & $76.86^{*}$ \\
\hline
\end{tabular}

Note: ${ }^{*}$ indicates $p<0.001$.

Table 4. Classification Accuracy (\%) for the Peer Evaluation

\begin{tabular}{lc|lc}
\hline Workload & \multirow{2}{*}{ Training } & \multicolumn{2}{|c}{ Workload Condition } \\
\cline { 3 - 4 } & & Low & High \\
\hline \multirow{3}{*}{ Cognitive } & SUP & 80.58 & 95.79 \\
& PEER & $\mathbf{9 7 . 0 5}$ & 94.52 \\
& BOTH & 92.12 & $\mathbf{9 8 . 3 1}$ \\
\hline \multirow{3}{*}{ Physical } & SUP & 67.90 & 58.88 \\
& PEER & $\mathbf{9 0 . 5 0}$ & $\mathbf{9 0 . 3 5}$ \\
& BOTH & 83.78 & 79.00 \\
\hline \multirow{3}{*}{ Overall } & SUP & 84.27 & 96.60 \\
& PEER & $\mathbf{9 4 . 7 8}$ & 96.55 \\
& BOTH & 93.84 & $\mathbf{9 7 . 0 4}$ \\
\hline
\end{tabular}

Bold represents highest accuracy per column.

5.1.1 Peer-based Evaluation Results. The peer-based evaluation contains low- and highworkload conditions. The IMPRINT Pro's modeled workload values and the algorithms' estimates for each workload condition are presented in Table 3. The PEER- and BOTH-trained algorithms' estimates are within a standard deviation of the IMPRINT Pro workload model values for each workload component. The SUP algorithm generally overestimated workload, due to the supervisory workload model values having a wider range, from minimum to maximum. The Kruskal-Wallis test determined that each trained algorithm's workload estimates significantly differed between workload conditions, except for the SUP algorithm's physical workload estimates.

A workload assessment algorithm must be able to detect various workload states to be viable for adaptive teaming systems. The algorithms' classification accuracy for low- and high-workload conditions is presented in Table 4. The PEER-trained algorithm achieves $>90 \%$ classification accuracy for each workload component. The BOTH and SUP algorithms classify cognitive and overall workload correctly at least $80 \%$ of the time, but both algorithms achieve lower physical workload accuracy. The SUP algorithm's physical workload accuracy is $<70 \%$, due to the algorithm receiving 
Table 5. Classification Accuracy (\%) by Peer-based Task

\begin{tabular}{ll|llllllll}
\hline \multirow{2}{*}{ Workload } & \multirow{2}{*}{ Training } & \multicolumn{7}{|c}{ Peer Evaluation Task } \\
\cline { 3 - 10 } Cognitive & $T 1_{L}$ & $T 1_{H}$ & $T 2_{L}$ & $T 2_{H}$ & $T 3_{L}$ & $T 3_{H}$ & $T 4_{L}$ & $T 4_{H}$ \\
\hline & SUP & 93 & 89 & 84 & 96 & 71 & 96 & 72 & $\mathbf{1 0 0}$ \\
& PEER & $\mathbf{1 0 0}$ & $\mathbf{9 3}$ & $\mathbf{9 5}$ & 94 & $\mathbf{9 6}$ & $\mathbf{1 0 0}$ & $\mathbf{9 6}$ & 89 \\
& BOTH & 98 & $\mathbf{9 3}$ & 92 & $\mathbf{1 0 0}$ & 86 & $\mathbf{1 0 0}$ & 90 & $\mathbf{1 0 0}$ \\
\hline \multirow{3}{*}{ Physical } & SUP & 78 & 60 & 61 & 38 & 65 & 61 & 64 & 70 \\
& PEER & $\mathbf{9 6}$ & $\mathbf{7 8}$ & $\mathbf{9 3}$ & $\mathbf{8 9}$ & $\mathbf{8 8}$ & $\mathbf{1 0 0}$ & $\mathbf{8 2}$ & $\mathbf{9 3}$ \\
& BOTH & $\mathbf{9 6}$ & 68 & 88 & 71 & 78 & 83 & 71 & 91 \\
\hline \multirow{3}{*}{ Overall } & SUP & 94 & 89 & 89 & 96 & 77 & $\mathbf{1 0 0}$ & 75 & $\mathbf{1 0 0}$ \\
& PEER & $\mathbf{9 8}$ & $\mathbf{9 3}$ & $\mathbf{9 2}$ & 92 & $\mathbf{8 8}$ & $\mathbf{1 0 0}$ & $\mathbf{1 0 0}$ & $\mathbf{1 0 0}$ \\
& BOTH & $\mathbf{9 8}$ & $\mathbf{9 3}$ & $\mathbf{9 2}$ & $\mathbf{9 8}$ & 84 & 96 & $\mathbf{1 0 0}$ & $\mathbf{1 0 0}$ \\
\hline
\end{tabular}

no prior training on the peer-based data. There is a negligible difference between the PEER- and BOTH-trained algorithms when classifying overall workload. It is interesting that the PEER algorithm achieves the lowest cognitive workload accuracy for the high-workload condition, which is due to the PEER algorithm underestimating high cognitive workload. Underestimating highworkload conditions means that the algorithm's estimates will be closer to the threshold between low and high workload, which decreases classification accuracy.

The peer evaluation workload conditions can be decomposed by task $(T 1-T 4)$ and workload condition $(L$ or $H)$. The classification accuracy by peer evaluation task and workload condition is provided in Table 5. The PEER algorithm achieves the highest physical workload classification accuracy for each task-workload condition pair, while achieving the highest classification accuracy for six of the cognitive workload task-workload condition pairs and seven of the overall workload task-workload condition pairs. The BOTH algorithm achieves the second-highest classification accuracy for each workload component, which is expected. The PEER- and BOTH-trained algorithms achieve $>80 \%$ accuracy when classifying cognitive or overall workload, with a negligible difference between the algorithms' overall workload classification accuracy for five tasks. The SUP algorithm achieves $>80 \%$ accuracy for six of the cognitive and all overall task-workload condition pairs. The SUP algorithm has poor physical workload classification.

The algorithms' tracking of workload shifts is analyzed using the Pearson's Correlation Coefficient. The correlation coefficients between the algorithms' estimates and workload models within and across the workload conditions for the entire evaluation are presented in Table 6. The Across column shows that each trained algorithm produced significant correlations across workload conditions, meaning that each algorithm can track large workload shifts (e.g., a shift from low to high workload). The significant correlations in the Within columns indicate that each algorithm can also track small workload shifts (i.e., a change in workload within the low-workload condition). The PEER-trained algorithm produced the highest correlations, followed by the BOTH algorithm.

It is not surprising that the PEER-trained algorithm produced the best results. The BOTH algorithm also produced very good results, even when incorporating the supervisory training data. The SUP algorithm performance is acceptable for the cognitive and overall workload assessments but is unable to accurately assess physical workload for the peer evaluation.

5.1.2 Supervisory-based Evaluation Results. The IMPRINT Pro modeled workload values and the algorithms' estimates for each supervisory condition (underload (UL), normal load (NL), and overload (OL)) are presented in Table 7. The SUP- and BOTH-trained algorithms' estimates are within one standard deviation of each IMPRINT Pro workload model for each workload 
Table 6. Peer Evaluation Correlation Coefficients for Within and Across Workload Conditions

\begin{tabular}{lc|cc|c}
\hline \multirow{2}{*}{ Workload } & \multirow{2}{*}{ Training } & \multicolumn{2}{|c|}{ Within } & \multirow{2}{*}{ Across } \\
\cline { 3 - 4 } Cognitive & SUP & $0.73^{*}$ & $0.58^{*}$ & $0.73^{*}$ \\
& PEER & $0.95^{*}$ & $0.85^{*}$ & $0.94^{*}$ \\
& BOTH & $0.79^{*}$ & $0.55^{*}$ & $0.74^{*}$ \\
\hline \multirow{3}{*}{ Physical } & SUP & $0.80^{*}$ & $0.63^{*}$ & $0.74^{*}$ \\
& PEER & $0.91^{*}$ & $0.92^{*}$ & $0.92^{*}$ \\
& BOTH & $0.87^{*}$ & $0.64^{*}$ & $0.79^{*}$ \\
\hline \multirow{2}{*}{ Overall } & SUP & $0.84^{*}$ & $0.54^{*}$ & $0.82^{*}$ \\
& PEER & $0.97^{*}$ & $0.91^{*}$ & $0.97^{*}$ \\
& BOTH & $0.93^{*}$ & $0.44^{*}$ & $0.83^{*}$ \\
\hline
\end{tabular}

Table 7. Workload Modeled and Algorithm Estimated Descriptive Statistics and Kruskal-Wallis for the Supervisory Evaluation

\begin{tabular}{lc|c|c|c|c}
\hline \multirow{2}{*}{ Workload } & \multirow{2}{*}{ Training } & \multicolumn{4}{|c}{ Workload Condition } \\
\cline { 3 - 6 } & & UL & NL & OL & $\chi^{2}$ \\
\hline \multirow{4}{*}{ Cognitive } & Model & $1.07(0.21)$ & $8.23(2.11)$ & $22.01(0.44)$ & $80.86^{*}$ \\
\cline { 2 - 6 } & SUP & $0.59(0.19)$ & $8.37(2.12)$ & $22.15(1.09)$ & $79.12^{*}$ \\
& PEER & $2.35(0.11)$ & $5.52(1.09)$ & $6.95(0.03)$ & $76.57^{*}$ \\
& BOTH & $0.46(0.43)$ & $7.68(2.52)$ & $20.22(1.68)$ & $78.91^{*}$ \\
\hline \multirow{5}{*}{ Physical } & Model & $0.12(0.25)$ & $4.61(2.03)$ & $11.76(0.43)$ & $80.38^{*}$ \\
& SUP & $0.11(0.26)$ & $4.99(2.10)$ & $11.84(0.38)$ & $80.46^{*}$ \\
& PEER & $0.18(0.45)$ & $3.38(1.22)$ & $3.91(0.07)$ & $58.75^{*}$ \\
& BOTH & $0.25(0.59)$ & $4.53(2.15)$ & $11.18(0.68)$ & $77.23^{*}$ \\
\hline \multirow{5}{*}{ Overall } & Model & $4.53(1.97)$ & $27.21(9.36)$ & $63.19(4.73)$ & $80.00^{*}$ \\
& SUP & $4.04(0.91)$ & $27.66(6.08)$ & $63.25(1.39)$ & $79.12^{*}$ \\
& PEER & $5.87(0.85)$ & $23.19(4.56)$ & $40.01(1.15)$ & $79.12^{*}$ \\
& BOTH & $4.05(1.30)$ & $26.51(5.64)$ & $60.66(2.58)$ & $79.12^{*}$ \\
\hline
\end{tabular}

component. The PEER-trained algorithm overestimates the underload condition and underestimates the normal load and overload conditions for each workload component. The Kruskal-Wallis test determined that the algorithms' estimates significantly differed between conditions for each workload component.

The workload assessment algorithm was designed to classify various workload levels. The algorithms' supervisory classification accuracies are presented in Table 8. Each trained algorithm achieves a $100 \%$ classification accuracy for the underload condition and has $\geq 85 \%$ accuracy for the normal load condition for each workload component. The SUP and BOTH algorithms achieve $\geq 90 \%$ classification accuracy for the overload condition, while the PEER algorithm is unable to classify the condition due to the PEER training data's maximum value being lower than the threshold (49.20, Table 2 ) between the normal load-overload task condition pair. The supervisory evaluation cannot be easily decomposed into separate tasks that permits analysis of the individual tasks for the classification accuracy; thus, such results are not presented.

The algorithms' ability to track workload shifts is analyzed using the Pearson's correlation coefficient analysis. The correlations for within and across workload conditions are presented in 
Table 8. Classification Accuracy (\%) for the Supervisory Eval

\begin{tabular}{lc|ccc}
\hline \multirow{2}{*}{ Workload } & \multirow{2}{*}{ Training } & \multicolumn{3}{|c}{ Workload Condition } \\
\cline { 2 - 5 } Cognitive & SUP & $\mathbf{1 0 0}$ & 93.74 & OL \\
\hline & PEER & $\mathbf{1 0 0}$ & 89.37 & 0 \\
& BOTH & $\mathbf{1 0 0}$ & $\mathbf{9 4 . 9 6}$ & 98.33 \\
\hline \multirow{3}{*}{ Physical } & SUP & $\mathbf{1 0 0}$ & 95.53 & $\mathbf{1 0 0}$ \\
& PEER & $\mathbf{1 0 0}$ & $\mathbf{9 6 . 6 4}$ & 0 \\
& BOTH & $\mathbf{1 0 0}$ & 93.85 & $\mathbf{1 0 0}$ \\
\hline \multirow{3}{*}{ Overall } & SUP & $\mathbf{1 0 0}$ & $\mathbf{1 0 0}$ & $\mathbf{1 0 0}$ \\
& PEER & $\mathbf{1 0 0}$ & $\mathbf{1 0 0}$ & 0 \\
& BOTH & $\mathbf{1 0 0}$ & $\mathbf{1 0 0}$ & $\mathbf{1 0 0}$ \\
\hline
\end{tabular}

Table 9. Supervisory Evaluation's Correlations for Within and Across Workload Conditions

\begin{tabular}{lc|ccc|c}
\hline \multirow{2}{*}{ Workload } & \multirow{2}{*}{ Training } & \multicolumn{3}{|c|}{ Within } & \multirow{2}{*}{ Across } \\
\cline { 3 - 5 } & & UL & NL & OL & \\
\hline \multirow{3}{*}{ Cognitive } & SUP & -0.07 & $0.45^{*}$ & -0.04 & $0.99^{*}$ \\
& PEER & $-0.59^{*}$ & -0.02 & -0.44 & $0.86^{*}$ \\
& BOTH & 0.16 & 0.17 & $0.67^{*}$ & $0.97^{*}$ \\
\hline \multirow{3}{*}{ Physical } & SUP & $0.92^{*}$ & $0.85^{*}$ & 0.19 & $0.98^{*}$ \\
& PEER & $0.91^{*}$ & $0.86^{*}$ & $0.65^{*}$ & $0.83^{*}$ \\
& BOTH & $0.91^{*}$ & $0.84^{*}$ & 0.02 & $0.98^{*}$ \\
\hline \multirow{2}{*}{ Overall } & SUP & $0.96^{*}$ & $0.89^{*}$ & $0.53^{*}$ & $0.99^{*}$ \\
& PEER & $0.95^{*}$ & $0.93^{*}$ & $0.96^{*}$ & $0.98^{*}$ \\
& BOTH & $0.83^{*}$ & $0.84^{*}$ & $0.79^{*}$ & $0.99^{*}$ \\
\hline
\end{tabular}

Table 9. The significant correlations in the Across column indicate that the algorithms can track large workload shifts; however, each trained algorithm has difficulty tracking small workload shifts in the underload and overload conditions for the cognitive workload component. The negative cognitive workload correlations are due to the static nature of the IMPRINT Pro workload models. A human's physiological signals typically oscillate, resulting in oscillating workload estimates; thus, the estimates oscillate around the static workload model values, resulting in negative correlations.

The SUP algorithm performs the best, followed by the BOTH algorithm for the supervisory relationship. The PEER algorithm performs well, except when classifying the overload state.

5.1.3 Workload Assessment Algorithm Discussion. Real-world dynamic domains, such as first response, contain multiple tasks with varying workload compositions. It is essential that a workload assessment algorithm classify workload for each task within the domain to be viable in realworld dynamic domains, which requires assessing overall workload and each workload component. The state-of-the-art algorithms typically only assess cognitive workload [24]; thus, limiting the algorithms to cognitively focused tasks. The presented workload assessment algorithm overcomes this limitation by estimating overall workload and each workload component. The current effort limits the workload components to cognitive and physical workload.

$H_{1}$ evaluates the algorithms' ability to classify cognitive, physical, and overall workload by stating that each algorithm will correctly classify workload states $\geq 80 \%$ of the time when trained 
and tested on data from the same evaluation. The hypothesis was upheld for the SUP and PEER algorithms, when the algorithms are classifying data from their corresponding evaluation (i.e., the PEER algorithm classifying the peer evaluation's data). The hypothesis was not upheld for the BOTH algorithm for the peer evaluation's physical workload component, which indicates that the supervisory data negatively impacts the classification accuracy. However, the BOTH algorithm achieves a $79 \%$ physical workload classification accuracy, which is slightly below the threshold.

The long-term objective is to develop an adaptive workload system that generalizes across human-robotic interaction paradigms; thus, $\mathrm{H}_{2}$ evaluates the algorithms' classification accuracy across interaction paradigms. The hypothesis was partially supported. The peer evaluation's SUP algorithm achieves $>80 \%$ classification accuracy when classifying cognitive and overall workload but achieves poor classification for physical workload. Likewise, the supervisory evaluation's PEER algorithm achieves high classification accuracy for the underload and normal load conditions for overall workload and each workload component but cannot classify the overload condition due to the peer training data's maximum value being below the threshold value separating the normal load and overload conditions. A limitation is that the algorithm must be trained on a similar set of workload conditions to generalize across human-robotic interaction paradigms.

$\mathrm{H}_{3}$ evaluated the ability to track workload shifts and was supported for all algorithms when using the peer evaluation dataset. The supervisory dataset analysis only provided partial support, as large workload shifts were tracked, but not small shifts in the underload and overload conditions. The underload and overload models are typically static (low variance), due to limits on the models (e.g., the underload model cannot go below zero). However, a human's physiological signals can oscillate; thus, the algorithms' estimates fluctuate around the static models, resulting in low correlations.

The developed workload assessment algorithm typically achieves the highest accuracy when using data from the same interaction paradigm. However, the BOTH algorithm that incorporates an equal number of data points from both datasets does achieve the highest accuracy in some cases, demonstrating the benefit of training using both datasets. The BOTH algorithm's high accuracy indicates the potential for using a single algorithm for both types of tasks and interaction paradigms. However, it is uncertain how the algorithm will perform in other human-robot interaction paradigms, beyond the evaluated paradigms.

\subsection{Task Generalizability Analysis}

It is important that a workload assessment algorithm be capable of generalizing across tasks within a human-robot teaming paradigm, as it is infeasible to train an adaptive teaming system on every task a human may complete within the teaming paradigm. Only data from the peer-based evaluation were used to assess the algorithm's task generalizability, as the supervisory evaluation's tasks are concurrent and not easily separated. The workload assessment algorithm was cross-validated using a leave-one-task-out approach [7], which creates four trained algorithms. Each algorithm was trained on three peer-based tasks using data from all 18 peer-based participants. The testing set was the data from all 18 participants for the peer-based task that was not used for training. Individual differences may not affect the results, as data from each participant were used to train each algorithm. The thresholds for classifying the workload conditions are the same as Table 2 .

It is expected that classification accuracy will decrease when the algorithm is not trained on a specific peer-based task; thus, hypothesis $\mathrm{H}_{4}$ predicts that the algorithm will correctly classify workload states for each workload component and overall workload at least $70 \%$ of the time when trained on three peer-based tasks and tested on the remaining task. Although classification accuracy will decrease, it is expected that each trained algorithm will track workload shifts for an 
Table 10. Generalized Task Estimates and Model Values by Peer Task and Workload Component

\begin{tabular}{l|c|c|c|c|c|c}
\hline \multirow{2}{*}{ Task } & \multicolumn{2}{|c|}{ Cognitive Workload } & \multicolumn{2}{c|}{ Physical Workload } & \multicolumn{2}{c}{ Overall Workload } \\
\cline { 2 - 7 } & Estimate & Model & Estimate & Model & Estimate & Model \\
\hline$T 1_{L}$ & $3.03(1.53)$ & $3.61(2.48)$ & $5.27(3.59)$ & $3.61(2.48)$ & $15.17(6.11)$ & $14.92(7.12)$ \\
$T 1_{H}$ & $4.36(0.54)$ & $5.79(1.08)$ & $6.77(1.74)$ & $5.79(1.08)$ & $19.72(1.8)$ & $20.62(3.07)$ \\
\hline$T 2_{L}$ & $1.77(2.16)$ & $1.78(2.29)$ & $4.07(2.82)$ & $1.78(2.29)$ & $11.82(5.57)$ & $11.78(2.29)$ \\
$T 2_{H}$ & $4.06(2.12)$ & $5.26(2.67)$ & $5.23(0.87)$ & $5.26(2.67)$ & $18.85(2.07)$ & $19.35(3.47)$ \\
$T 3_{L}$ & $3.75(1.52)$ & $3.85(3.02)$ & $3.31(2.74)$ & $3.85(3.02)$ & $13.11(7.31)$ & $12.28(9.5)$ \\
$T 3_{H}$ & $4.95(0.34)$ & $6.49(0.96)$ & $4.08(1.96)$ & $6.49(0.96)$ & $19.06(1.81)$ & $20.53(2.83)$ \\
\hline$T 4_{L}$ & $3.05(2.09)$ & $3.67(3.05)$ & $4.68(2.4)$ & $3.67(3.05)$ & $13.49(8.41)$ & $15.17(9.61)$ \\
$T 4_{H}$ & $4.92(0.55)$ & $6.49(0.97)$ & $6.16(1.35)$ & $6.49(0.97)$ & $21.11(1.36)$ & $24.00(2.94)$ \\
\hline
\end{tabular}

Table 11. Generalized Task Classification Accuracy (\%) by Peer Task and Workload Component

\begin{tabular}{l|cccccccc}
\hline \multirow{2}{*}{ Workload } & \multicolumn{8}{|c}{ Peer Evaluation Task } \\
\cline { 2 - 9 } & $T 1_{L}$ & $T 1_{H}$ & $T 2_{L}$ & $T 2_{H}$ & $T 3_{L}$ & $T 3_{H}$ & $T 4_{L}$ & $T 4_{H}$ \\
\hline Cognitive & 78.32 & 74.79 & 93.22 & 100.00 & 88.83 & 96.04 & 88.82 & 89.29 \\
Physical & 89.51 & 73.08 & 73.45 & 90.78 & 85.11 & 100.00 & 62.94 & 70.83 \\
Overall & 96.50 & 93.16 & 90.40 & 88.83 & 81.91 & 100.00 & 99.41 & 100.00 \\
\hline
\end{tabular}

Table 12. Generalized Task Pearson's Correlation Coefficients by Peer Task and Workload Component

\begin{tabular}{l|cccccccc}
\hline \multirow{2}{*}{ Workload } & \multicolumn{7}{|c}{ Peer Evaluation Task } \\
\cline { 2 - 8 } & $T 1_{L}$ & $T 1_{H}$ & $T 2_{L}$ & $T 2_{H}$ & $T 3_{L}$ & $T 3_{H}$ & $T 4_{L}$ & $T 4_{H}$ \\
\hline Cognitive & $0.95^{*}$ & $0.70^{*}$ & $0.97^{*}$ & $0.95^{*}$ & $0.82^{*}$ & $0.48^{*}$ & $0.82^{*}$ & $0.48^{*}$ \\
Physical & $0.98^{*}$ & $0.85^{*}$ & $0.41^{*}$ & $0.63^{*}$ & $0.80^{*}$ & $0.96^{*}$ & $0.64^{*}$ & $0.88^{*}$ \\
Overall & $0.98^{*}$ & $0.88^{*}$ & $0.89^{*}$ & $0.95^{*}$ & $0.96^{*}$ & $0.92^{*}$ & $0.96^{*}$ & $0.89^{*}$ \\
\hline
\end{tabular}

unforeseen task. Hypothesis $\mathrm{H}_{5}$ predicts that each trained algorithm's estimates will significantly and positively correlate with the IMPRINT Pro workload models for each peer-based task.

5.2.1 Task Generalizability Results. Only the results for the algorithms' performance on each testing set are presented. For example, a result for $T 1_{L}$ represents the testing set performance for the algorithm trained on tasks two, three, and four for both workload conditions (low (L) and high $(\mathrm{H})$ ). The algorithms' estimates and model's mean and standard deviation's are presented in Table 10. The algorithm underestimated cognitive workload for the high-workload tasks and overestimated physical workload for the low-workload tasks. The algorithm's overall workload estimates were close to the model's values.

Correctly classifying workload for unforeseen tasks is essential to an adaptive teaming system, as it is infeasible to collect data for all tasks. The algorithms' classification accuracies by task and workload component are provided in Table 11 . The algorithms correctly classify workload $\geq 70 \%$, except for $T 4_{L}$, due to the PEER algorithm having low physical workload classification accuracy.

The algorithm's ability to track workload shifts for each task is analyzed using the Pearson's correlation coefficient, which are presented in Table 12. Each algorithms' estimates significantly correlates with each corresponding model, which demonstrates that the algorithms' estimates correctly reflects workload shifts for unforeseen tasks. 
5.2.2 Task Generalizability Discussion. A workload assessment algorithm's ability to generalize across tasks within the same human-robot teaming paradigm is essential for an adaptive teaming system, as it is difficult to collect training data for each task within a paradigm. Hypothesis $\mathrm{H}_{4}$ focused on evaluating the algorithm's task generalizability and is supported for cognitive and overall workload classification. The hypothesis is only partially supported for physical workload classification, as the algorithm fails to correctly classify physical workload for $T 4_{L}$ at least $70 \%$ of the time. This result is due to task $T 4_{L}$ substantially differing from the other peer-based tasks. Partially supporting hypothesis $\mathrm{H}_{4}$ for physical workload classification is not detrimental to the algorithm's task generalizability, as the average physical workload accuracy is above $70 \%$. Further, none of the state-of-the-art workload assessment algorithms generalize across tasks; thus, showing that the algorithm generalizes across tasks for cognitive and overall workload, a significant contribution.

The workload assessment algorithm's estimates must accurately reflect a shift in workload regardless of the task, which represents hypothesis $\mathrm{H}_{5}$. This hypothesis is upheld, as the algorithm's estimates significantly correlate to the workload model values for each task. Having high confidence that a shift in the algorithm's estimates correctly represents a shift in workload allows an adaptive teaming system to better gauge the affect an adaptation has on the human's workload state. For example, if the human's is overloaded and the workload estimates show the workload level is decreasing appropriately, then the adaptive system can be confident that no adaptation is needed.

The task generalizability analysis demonstrates the workload assessment algorithm's ability to generalize across similar tasks for the PEER dataset. However, it is unclear whether the algorithm can generalize across tasks for other human-robot teaming domains or what happens if an unforeseen task differs substantially from the tasks on which the algorithm was trained.

\subsection{Cross-Interaction Paradigm Analysis}

The peer-based evaluation included human-robot and human-human teaming scenarios, which allows for analyzing the impact that training with additional workload data from the humanhuman teaming interaction paradigm has. The IMPRINT Pro model results indicate that the human-human teaming tended to have lower workload than the human-robot scenario, but a Kruskal-Wallis test found no significant differences. This analysis focuses solely on the peer-based relationship, as the supervisory-based evaluation did not include human-human teams. Three algorithms were trained: only human-human data $(\mathrm{HH})$, both the human-human and humanrobot data (HH-HR), and only the human-robot data (PEER), the same dataset as in Section 5.1. Each algorithm was trained using data from 12 peer-evaluation participants, while data from 6 peer-evaluation H-R participants were used for testing. The workload classification thresholds are provided in Table 2 .

It is expected the the human-human teaming data will minimally impact the algorithm's classification accuracy for human-robot teams; thus, hypothesis $\mathrm{H}_{6}$ predicts that the HH-HR algorithm's classification accuracy will be within 5\% of the PEER algorithm's accuracy. The hypothesis also predicts that the $\mathrm{HH}$ algorithm's classification accuracy will be within $5 \%$ of the PEER algorithm's accuracy, as there is no significant difference between the IMPRINT Pro models for each teaming scenario. Further, it is expected that the HH-HR and HH algorithms will be able to track workload shifts. Hypothesis $\mathrm{H}_{7}$ predicts that both algorithm's workload estimates will significantly and positively correlate with the workload models for each peer-based task.

5.3.1 Cross-Interaction Paradigm Results. The workload assessment algorithm's crossinteraction paradigm performance is examined by comparing the algorithms' estimates to the 
Table 13. Descriptive and Kruskall-Wallis Statistics for the IMPRINT Pro Workload Model Values and the $\mathrm{HH}, \mathrm{HH}-\mathrm{HR}$, and PEER Algorithms' Workload

Estimates for the Peer-based Evaluation

\begin{tabular}{lc|c|c|c}
\hline \multirow{2}{*}{ Workload } & \multirow{2}{*}{ Training } & \multicolumn{3}{|c}{ Workload Condition } \\
\cline { 3 - 5 } & & Low & High & $\chi^{2}$ \\
\hline \multirow{2}{*}{ Cognitive } & Model & $3.58(2.84)$ & $6.14(1.59)$ & $61.75^{*}$ \\
\cline { 2 - 5 } & HH & $3.49(2.48)$ & $8.68(1.22)$ & $44.02^{*}$ \\
& HH-HR & $3.45(2.42)$ & $5.50(1.14)$ & $59.15^{*}$ \\
& PEER & $3.77(2.82)$ & $5.95(1.40)$ & $35.92^{*}$ \\
\hline \multirow{2}{*}{ Physical } & Model & $4.17(2.63)$ & $5.56(2.14)$ & $17.85^{*}$ \\
& HH & $4.00(2.42)$ & $6.02(1.88)$ & $17.51^{*}$ \\
& HH-HR & $4.07(6.02)$ & $6.02(1.82)$ & $15.00^{*}$ \\
& PEER & $4.28(2.50)$ & $5.35(1.89)$ & $10.94^{*}$ \\
\hline \multirow{2}{*}{ Overall } & Model & $14.33(7.83)$ & $21.31(3.36)$ & $56.26^{*}$ \\
& HH & $13.63(7.51)$ & $20.97(2.41)$ & $72.25^{*}$ \\
& HH-HR & $13.65(7.51)$ & $20.78(2.51)$ & $62.34^{*}$ \\
& PEER & $14.64(7.62)$ & $20.91(2.25)$ & $45.60^{*}$ \\
\hline
\end{tabular}

Table 14. HH-, HH-HR-, and PEER-Trained Algorithms' Classification Accuracy

\begin{tabular}{lc|c|c}
\hline \multirow{2}{*}{ Workload } & \multirow{2}{*}{ Training } & \multicolumn{2}{|c}{ Workload Condition } \\
\cline { 3 - 4 } & & Low & High \\
\hline Cognitive & HH & 94.92 & 94.63 \\
& HH-HR & $\mathbf{9 7 . 1 4}$ & $\mathbf{9 6 . 2 3}$ \\
& PEER & 97.05 & 94.52 \\
\hline Physical & HH & 88.25 & 86.55 \\
& HH-HR & 87.30 & 86.02 \\
& PEER & $\mathbf{9 0 . 5 0}$ & $\mathbf{9 0 . 3 5}$ \\
\hline Overall & HH & 92.69 & $\mathbf{9 6 . 7 7}$ \\
& HH-HR & 92.40 & 95.70 \\
& PEER & $\mathbf{9 4 . 7 8}$ & 96.55 \\
\hline
\end{tabular}

Bold represents highest accuracy per column.

IMPRINT Pro modeled workload values for the peer-based human-robot teaming tasks. The algorithms' estimates are provided in Table 13. The HH-HR algorithm's estimates are within a standard deviation of the IMPRINT Pro workload model values for each workload component. The HH algorithm's physical and overall workload estimates are within a standard deviation of the IMPRINT Pro values, but the algorithm's workload estimates for high cognitive workload are not. The HH and HH-HR algorithms tend to overestimate high physical workload and underestimate low workload but do so minimally. The Kruskal-Wallis test found that each algorithms' estimates significantly differed between workload conditions. All three algorithm's estimates are within $10 \%$ of each other, illustrating that the respective trained algorithm produces accurate workload estimates.

Each algorithm must accurately classify workload for the peer-based evaluation's H-R teaming scenario. The algorithms' classification accuracies are presented in Table 14. The HH-HR algorithm achieves high classification accuracy for overall workload and its components. However, the 
Table 15. HH, HH-HR, and PEER Algorithms' Correlation Coefficients for Within and Across Workload Conditions

\begin{tabular}{lc|l|l|l}
\hline Workload & \multirow{2}{*}{ Training } & \multicolumn{2}{|c|}{ Within } & \multirow{2}{*}{ Across } \\
\cline { 3 - 4 } & & Low & High & \\
\hline Cognitive & HH & $0.93^{*}$ & $0.81^{*}$ & $0.93^{*}$ \\
& HH-HR & $0.95^{*}$ & $0.83^{*}$ & $0.90^{*}$ \\
& PEER & $0.95^{*}$ & $0.85^{*}$ & $0.94^{*}$ \\
\hline Physical & HH & $0.87^{*}$ & $0.91^{*}$ & $0.91^{*}$ \\
& HH-HR & $0.87^{*}$ & $0.90^{*}$ & $0.94^{*}$ \\
& PEER & $0.91^{*}$ & $0.92^{*}$ & $0.92^{*}$ \\
\hline Overall & HH & $0.97^{*}$ & $0.91^{*}$ & $0.97^{*}$ \\
& HH-HR & $0.97^{*}$ & $0.91^{*}$ & $0.98^{*}$ \\
& PEER & $0.97^{*}$ & $0.91^{*}$ & $0.97^{*}$ \\
\hline
\end{tabular}

HH-HR and the HH algorithms' physical workload classification accuracy is approximately $4 \%$ lower than the PEER algorithm's accuracy, which demonstrates that the H-H teaming data negatively impacts the HH-HR algorithm's classification accuracy. The HH-HR algorithm's cognitive and overall classification accuracy are within $2 \%$ of the PEER algorithm's accuracy.

The Pearson's correlation coefficients between the algorithms' estimates and IMPRINT Pro models are used to analyze the algorithms' ability to track workload shifts within and across workload conditions. The correlation coefficients are presented in Table 15. The algorithm's estimates significantly correlate with the IMPRINT Pro workload models, demonstrating that each algorithm tracks workload shifts across and within workload conditions. The correlation coefficients for each algorithm are similar to each other, thus, illustrating that the $\mathrm{H}-\mathrm{H}$ teaming data do not negatively impact the algorithm's ability to track workload shifts.

5.3.2 Cross-Interaction Paradigm Discussion. Incorporating human-human teaming data can increase the amount of available training data, but it must have a minimal impact on an adaptive teaming system's ability to classify workload for human-robot teaming scenarios. Hypothesis $\mathrm{H}_{6}$ focuses on such impacts to the algorithm's classification accuracy by stating that the HH-HR and HH-trained algorithms' accuracy will be within 5\% of the PEER-trained algorithm's accuracy. The hypothesis is fully supported, which demonstrates that the human-human teaming data do not significantly impact the algorithm's classification accuracy for human-robot teaming paradigms. Further, the algorithm trained only on human-human teaming data still achieved high performance for human-robot teaming scenarios, as the two scenarios have similar workload levels.

The HH-HR-trained algorithm also needs to track workload shifts within and across workload conditions. Hypothesis $\mathrm{H}_{7}$ states that the $\mathrm{HH}$ and $\mathrm{HH}-\mathrm{HR}$-trained algorithms' estimates will significantly correlate with the IMPRINT Pro workload models. The hypothesis is supported, which illustrates that incorporating human-human teaming data did not impact the algorithm's ability to track workload shifts within and across workload conditions. Similarly, an algorithm trained solely on human-human teaming data tracked workload shifts for a human-robot teaming scenario. Overall, the workload assessment algorithm trained on human-robot and human-human teaming datasets did not substantially decrease performance. Further, an algorithm trained on a human-human teaming scenario achieved high performance in a similar human-robot scenario.

\section{CONCLUSION}

The diagnostic workload assessment algorithm's ability to classify workload for multiple teaming paradigms, generalize across populations, and generalize across tasks is analyzed by training 
the algorithm on multiple datasets. The results highlight that the algorithm achieves high classification accuracy when trained and tested on data from the same human-robot teaming paradigm. Further, the algorithm generalized across the participant populations for each human-robot teaming evaluation. The algorithm's task generalizability was analyzed using tasks from the peer-based evaluation, which showed that the algorithm generalized across peer-based tasks for cognitive and overall workload estimation. The impact of training the algorithm on multiple datasets was also analyzed by training the algorithm on two human-robot teaming datasets and on the peer-based evaluation's human-human and human-robot datasets. The results demonstrated that there is minimal negative impact on the algorithm's performance.

A common theme among the algorithm analyses is that the workload assessment algorithm had some difficulty assessing physical workload for the peer-based evaluation, which is not due to the nature of the physical workload assessment but rather due to the algorithm's workload sensitivity. The algorithm had little difficulty discriminating between supervisory-based evaluations of workload conditions, as there were extremely large effect sizes between the conditions (Cohen's $\mathrm{d}>1.0$ ). Effect sizes were calculated using the IMPRINT Pro workload models. The algorithm's performance decreased for the peer-based evaluation, as the effect sizes were smaller. The effect sizes for the peer-based evaluation's cognitive and physical workload conditions are still considered large, while there is a medium effect size between the physical workload conditions. This effect size analysis shows that the algorithm is sensitive to large workload differences, but sensitivity decreases for smaller differences, which is not surprising. It is difficult to compare the algorithm's sensitivity to the state-of-the-art algorithms, as the other algorithms do not examine sensitivity. The state-of-the-art algorithms only present classification accuracy, which is dependent on the difference between workload conditions, but effect sizes cannot be extrapolated from classification accuracy.

The current algorithm relies on workload models to assess the visual, auditory, and speech workload components. The algorithm will be extended to estimate the auditory and speech workload components using appropriately sensitive workload metrics (e.g., noise level, speech rate, speech response time) that provide values for the respective workload equations. Visual workload is difficult to estimate in dynamic domains (e.g., first response), as eye-tracking metrics require known focus regions. Thus, for real-world dynamic domains, a visual workload model will continue to be used to assess this workload component, at least until appropriate technology is available. The current workload assessment algorithm assumes that the human's task is always known; however, this will not be the case for dynamic real-world domains. Such domains will require activity recognition to identify the humans' tasks; thus, an activity recognition algorithm (e.g., [16, 41, 49]) can be integrated to identify the current task focus.

Diagnostically assessing workload is imperative for the envisioned adaptive teaming system, as assessing overall workload and each workload component determines what distinct components are contributing to the overall workload state or simply why the human is in the current workload state. Identifying the distinct contributing components permits targeted adaptions to the components to normalize the workload state. An adaptation based solely on the overall workload state cannot target a specific workload component; thus, the adaptation may be ineffective (i.e., reallocating a task that is not a primary contributor to the workload state). A diagnostic workload assessment permits projection of future interactions or task allocations onto the human's current workload state to determine and account for the potential impact.

The presented workload assessment algorithm estimates the cognitive and physical workload components based on objective metrics (i.e., physiological signals) and uses models to estimate the auditory, visual, and speech workload components. The component workload estimates are combined into an overall workload value that is used to determine the human's workload state, and if 
task performance is likely to decrease due to the human's current state. The workload component values can be used to normalize the human's workload state by modifying a robot's interaction modality or the distribution of tasks across team members based on the distinct workload components. Data from two human-robotic interaction evaluations was used to evaluate the workload assessment algorithm's ability to classify workload, generalize across populations, and generalize across two human-robot teaming paradigms: peer and supervisory. Further investigation is need to determine the algorithm's performance on other human-robot teaming paradigms.

\section{REFERENCES}

[1] J. Aasman, G. Mulder, and L. Mulder. 1987. Operator effort and the measurement of heart-rate variability. Hum. Factors 29, 2 (1987), 161-170.

[2] Martín Abadi, Paul Barham, Jianmin Chen, Zhifeng Chen, Andy Davis, Jeffrey Dean, Matthieu Devin, Sanjay Ghemawat, Geoffrey Irving, Michael Isard, et al. 2016. Tensorflow: A system for large-scale machine learning. In Proceedings of the USENIX Symposium on Operating Systems Design and Implementation (OSDI'16), Vol. 16. $265-283$.

[3] H. A. Abbass, J. Tang, R. Amin, M. Ellejmi, and S. Kirby. 2014. Augmented cognition using real-time EEG-based adaptive strategies for air traffic control. In Proceedings of the Human Factors and Ergonomics Society Annual Meeting, Vol. 58. Sage Publications, 230-234.

[4] S. Archer, M. Gosakan, P. Shorter, and J. Lockett. 2005. New capabilities of the Armys maintenance manpower modeling tool. J. Int. Test Eval. Assoc. 26, 1 (2005), 19-26.

[5] R. W. Backs and L. C. Walrath. 1992. Eye movement and pupillary response indices of mental workload during visual search of symbolic displays. Appl. Ergonom. 23, 4 (1992), 243-254.

[6] A. Berthold and A. Jameson. 1999. Interpreting symptoms of cognitive load in speech input. In International Conference on User Modeling (1999), 235-244.

[7] C. M Bishop. 2006. Pattern Recognition. Vol. 128. Springer-Verlag, New York, NY.

[8] M. Brenner, E. Doherty, and T. Shipp. 1994. Speech measures indicating workload demand. Aviat. Space Environ. Med. 65, 1 (1994), 21-26.

[9] B. Cain. 2007. A Review of Mental Workoad Literature. Techreport RTO-TR-HFM-121-Part-II. Defence Research and Development Toronto.

[10] M. Castor. 2003. GARTEUR Handbook of Mental Workload Measurement. Group for Aeronautical Research and Technology in Europe.

[11] J. C. Christensen, J. R. Estepp, G. F. Wilson, and C. A. Russell. 2012. The effects of day-to-day variability of physiological data on operator functional state classification. NeuroImage 59, 1 (2012), 57-63.

[12] J. Raymond Comstock and R. J. Arnegard. 1992. The Multi-Attribute Task Battery for Operator Workload and Strategic Behavior Research. Technical Report NASA Tech. Memorandum 104174. NASA Langley Research Center.

[13] Aymeric Damien et al. 2016. TFLearn. Retrieved from https://github.com/tflearn/tflearn.

[14] K. Durkee, A. Hiriyanna, S. Pappada, J. Feeney, and S. Galster. 2016. Multi-model approach to human functional state estimation. In Lecture Notes in Computer Science. Springer-Verlag, Switzerland, 188-197.

[15] M. A. Goodrich and A. C. Schultz. 2007. Human-robot interaction: A survey. Found. Trends Hum.-Comput. Interact. 1 , 3 (2007), 203-275.

[16] I. Gori, J. K. Aggarwal, L. Matthies, and M. Ryoo. 2016. Multitype activity recognition in robot-centric scenarios. IEEE Robot. Automat. Lett. 1, 1 (2016), 593-600.

[17] Peter A. Hancock and James L. Szalma. 2008. Performance under Stress. Ashgate Publishing, Ltd.

[18] T. C. Hankins and G. F. Wilson. 1998. A comparison of heart rate, eye activity, EEG and subjective measures of pilot mental workload during flight. Aviat. Space Environ. Med. 69, 4 (1998), 360-367.

[19] C. E. Harriott. 2015. Workload and Task Performance in Human-Robot Peer-based Teams. Ph.D. Dissertation. Vanderbilt University.

[20] C. E. Harriott, T. Zhang, and J. A. Adams. 2013. Assessing physical workload for human-robot peer-based teams. Int. f. Hum.-Comput. Stud. 71, 7-8 (2013), 821-837.

[21] D. Harris. 2011. Human Performance on the Flight Deck. Ashgate Publishing Limited Surrey, U.K.

[22] S. G. Hart and L. E. Staveland. 1988. Development of NASA-TLX (task load index): Results of empirical and theoretical research. 52, 1 (1988), 139-183.

[23] J. Heard, C. E. Harriott, and J. A. Adams. 2017. A human workload assessment algorithm for collaborative humanmachine teams. In Proceedings of the IEEE International Symposium on Robot and Human Interactive Communication (RO-MAN'17). 366-371.

[24] J. Heard, C. E. Harriott, and J. A. Adams. 2018. A survey of workload assessment algorithms. IEEE Trans. Hum.-Mach. Syst. 48, 5 (2018), 434-451. 
[25] C. Herff, D. Heger, O. Fortmann, J. Hennrich, F. Putze, and T. Schultz. 2013. Mental workload during n-back taskquantified in the prefrontal cortex using fNIRS. Front. Hum. Neurosci. 7 (2013), 1-9.

[26] L. M. Hirshfield, E. Solovey, A. Girouard, J. Kebinger, R. Jacob, A. Sassaroli, and S. Fantini. 2009. Brain measurement for usability testing and adaptive interfaces: An example of uncovering syntactic workload with functional near infrared spectroscopy. In Proceedings of the SIGCHI Conference on Human Factors in Computing Systems. 2185-2194.

[27] R. Hoogendoorn and B. van Arem. 2013. Driver workload classification through neural network modeling using physiological indicators. In Proceedings of the IEEE Conference on Intelligent Transportation Systems. 2268-2273.

[28] C. Humphrey and J. A. Adams. 2011. Analysis of complex team-based systems: Augmentations to goaldirected task analysis and cognitive work analysis. Theor. Issues Ergonom. Sci. 12, 2 (2011), 149-175.

[29] ASTM International. 2010. Standard Guide for Operational Guidelines for Initial Response to a Suspected Biothreat Agent. Technical Report ASTM E2770-10. American Society for Testing and Materials.

[30] A. Jameson, J. Kiefer, C. Müller, B. Großmann-Hutter, F. Wittig, and R. Rummer. 2010. Assessment of a users time pressure and cognitive load on the basis of features of speech. In Resource-Adaptive Processes. Springer-Berlin, Heidelberg, 171-204.

[31] J. Johnstone, P. Ford, G. Hughes, T. Watson, and A. Garrett. 2012. BioHarness ${ }^{\text {TM }}$ multivariable monitoring device: Part. II: Reliability. J. Sports Sci. Med. 11, 3 (2012), 409.

[32] P.G. Jorna. 1993. Heart rate and workload variations in actual and simulated flight. Ergonomics 36, 9 (1993), 1043-1054.

[33] J. Keller, H. Bless, F. Blomann, and D. Kleinbohl. 2011. Physiological aspects of flow experiences: Skills-demandcompatibility effects on heart rate variability and salivary cortisol. F. Exp. Soc. Psychol. 47, 4 (2011), 849-852.

[34] D. Kingma and J. Ba. 2015. Adam: A method for stochastic optimization. In Proceedings of the International Conference on Learning Representations. 8-13.

[35] D. J. Lasley, R. D. Hamer, R. Dister, and T. E. Cohn. 1991. Postural stability and stereo-ambiguity in man-designed visual environments. IEEE Trans. Biomed. Eng. 38, 8 (1991), 808-813.

[36] G. Marquart, C. Cabrall, and J. de Winter. 2015. Review of eye-related measures of drivers' mental workload. Proc. Manufact. 3, 1 (2015), 2854-2861.

[37] J. McCraken and T. Aldrich. 1984. Implications of Operator Workload and System Automation Goals. Technical Report ASI-479-024-84B. U.S. Army Research Institution.

[38] D. Mitchell. 2000. Mental Workload and ARL Workload Modeling Tools. Technical Report ARL-TNL-161. Army Research Lab Aberden Proving Ground MD.

[39] S. Miyake, S. Yamada, T. Shoji, Y. Takae, N. Kuge, and T. Yamamura. 2009. Physiological responses to workload change. A test/retest examination. Appl. Ergonom. 40, 6 (Nov. 2009), 987-996.

[40] T. Mizuno, T. Sakai, S. Kawazura, H. Asano, K. Akehi, S. Matsuno, K. Mito, Y. Kume, and N. Itakura. 2016. Measuring facial skin temperature changes caused by mental work-load with infrared thermography. IEEE Trans. Electron. Inf Syst. 136, 11 (2016), 1581-1585.

[41] A. Nazábal, P. García-Moreno, A. Artés-Rodríguez, and Z. Ghahramani. 2016. Human activity recognition by combining a small number of classifiers. IEEE 7. Biomed. Health Inf. 20, 5 (2016), 1342-1351.

[42] R. Parasuraman. 2003. Neuroergonomics: Research and practice. Theor. Issues Ergonom. Sci. 4, 1-2 (2003), 5-20.

[43] P. Paul, F. M. Kuijer, B. Visser, and H. Kemper. 1999. Job rotation as a factor in reducing physical workload at a refuse collecting department. Ergonomics 42, 9 (1999), 1167-1178.

[44] D. Popovic, M. Stikic, T. Rosenthal, D. Klyde, and T. Schnell. 2015. Sensitive, diagnostic and multifaceted mental workload classifier (PHYSIOPRINT). In Foundations of Augmented Cognition, D.D. Schmorrow and C. M. Fidopiastis (Eds.). Springer-Verlag Switzerland, 101-111.

[45] F. Putze, J. Jarvis, and T. Schultz. 2010. Multimodal recognition of cognitive workload for multitasking in the car. In Proceedings of the IEEE Conference on Pattern Recognition. 3748-3751.

[46] B. Reimer, B. Mehler, J. Coughlin, K. Godfrey, and C. Tan. 2009. An on-road assessment of the impact of cognitive workload on physiological arousal in young adult drivers. In Proceedings of the 1st International Conference on Automotive User Interfaces and Interactive Vehicular Applications. 115-118.

[47] A. H. Roscoe. 1992. Assessing pilot workload. Why measure heart rate, HRV and respiration? Biol. Psychol. 34, 2-3 (1992), 259-287.

[48] C. Rusnock, B. Borghetti, and I. McQuaid. 2015. Objective-analytical measures of workload - the third pillar of workload triangulation? In Foundations of Augmented Cognition, Dylan D. Schmorrow and Cali M. Fidopiastis (Eds.). Springer Science. Switzerland, 124-135.

[49] M. S. Ryoo and L. Matthies. 2016. First-person activity recognition: Feature, temporal structure, and prediction. Int. f. Comput. Vis. 119, 3 (2016), 307-328.

[50] J. Scholtz. 2003. Theory and evaluation of human robot interactions. In Proceedings of the 36th Annual Hawaii International Conference on System Sciences. 10-16. 
[51] M. B. Sterman, D. A. Kaiser, C. A. Mann, B. Y. Suyenobu, D. C. Beyma, and J. R. Francis. 1993. Application of quantitative EEG analysis to workload assessment in an advanced aircraft simulator. In Proceedings of the Human Factors and Ergonomics Society Annual Meeting, Vol. 37. Sage Publications, 118-121.

[52] M. B. Sterman and C. A. Mann. 1995. Concepts and applications of EEG analysis in aviation performance evaluation. Biol. Psychol. 40, 1-2 (1995), 115-130.

[53] C. Ting, M. Mahfouf, A. Nassef, D. Linkens, G. Panoutsos, P. Nickel, A. Roberts, and G. Hockey. 2010. Real-time adaptive automation system based on identification of operator functional state in simulated process control operations. IEEE Trans. Syst. Man Cybernet. A 40, 2 (2010), 251-262.

[54] K. Vicente, D. Thornton, and N. Moray. 1987. Spectral analysis of sinus arrhythmia: A measure of mental effort. Hum. Factors 29, 2 (1987), 171-182.

[55] C. D. Wickens, J. D. Lee, Y. Liu, and S. E. Gordon Becker. 2004. An Introduction to Human Factors Engineering (2nd ed.). Pearson Education, Inc.

[56] Z. Yin and J. Zhang. 2014. Recognition of mental workload levels by combining adaptive exponential feature smoothing and locality preservation projection techniques. In Proceedings of the IEEE Chinese Control Conference. 4700-4705.

Received February 2018; revised November 2018; accepted February 2019 